\title{
Measurement of Rank and Other Properties of Direct and Scattered Signals
}

\author{
Svante Björklund, ${ }^{1}$ Per Grahn, ${ }^{1}$ Anders Nelander, ${ }^{1}$ and Mats I. Pettersson ${ }^{2}$ \\ ${ }^{1}$ The Swedish Defence Research Agency (FOI), P.O. Box 1165, 58111 Linköping, Sweden \\ ${ }^{2}$ The Blekinge Institute of Technology, 37179 Karlskrona, Sweden \\ Correspondence should be addressed to Svante Björklund; svabj@foi.se
}

Received 15 April 2016; Revised 12 July 2016; Accepted 25 July 2016

Academic Editor: Wenchong Xie

Copyright (C) 2016 Svante Björklund et al. This is an open access article distributed under the Creative Commons Attribution License, which permits unrestricted use, distribution, and reproduction in any medium, provided the original work is properly cited.

\begin{abstract}
We have designed an experiment for low-cost indoor measurements of rank and other properties of direct and scattered signals with radar interference suppression in mind. The signal rank is important also in many other applications, for example, DOA (Direction of Arrival) estimation, estimation of the number of and location of transmitters in electronic warfare, and increasing the capacity in wireless communications. In real radar applications, such measurements can be very expensive, for example, involving airborne radars with array antennas. We have performed the measurements in an anechoic chamber with several transmitters, a receiving array antenna, and a moving reflector. Our experiment takes several aspects into account: transmitted signals with different correlation, decorrelation of the signals during the acquisition interval, covariance matrix estimation, noise eigenvalue spread, calibration, near-field compensation, scattering in a rough surface, and good control of the influencing factors. With our measurements we have observed rank, DOA spectrum, and eigenpatterns of direct and scattered signals. The agreement of our measured properties with theoretic and simulated results in the literature shows that our experiment is realistic and sound. The detailed description of our experiment could serve as help for conducting other well-controlled experiments.
\end{abstract}

\section{Introduction}

In this article we have designed an experiment for low-cost indoor measurements of rank and other properties of direct and scattered signals with radar applications in mind. In real radar applications, such measurements can be very expensive, for example, involving airborne radars with array antennas.

In this introductory section we first in Section 1.1 define the signal rank and mention some other signal properties. Then in Section 1.2 we present radar applications and other applications where the signal rank is important. Section 1.3 tells what we have done in this article and gives an outline of the rest of the article.

1.1. Signal Rank and Other Signal Properties. In many applications of array antennas the covariance matrix $\mathbf{R}=E\left\{\mathbf{x} \mathbf{x}^{H}\right\}$ of the received signal vector $\mathbf{x}$ is utilized. The vector $\mathbf{x}$ usually contains the signals from the antenna channels and possibly some temporal dimension. The vector can be called a space (or space-only) snapshot or space-time snapshot, respectively.

The rank of the covariance matrix for the case with $\mathbf{x}$ containing only external signals and without the white receiver noise is important in radar applications and in many other applications (see Section 1.2). It states how many, in some sense, independent signals impinge on the antenna. We talk about the signal rank, which is the rank of this covariance matrix.

As $\mathbf{R}$ usually is unknown, it must be estimated in the algorithms that use it. A common estimate is $[1,2]$

$$
\widehat{\mathbf{R}}=\frac{1}{N_{R}} \sum_{n=1}^{N_{R}} \mathbf{x}_{n} \mathbf{x}_{n}{ }^{H},
$$

where $\mathbf{x}_{n}$ are training snapshots and $N_{R}$ is the number of such snapshots. These training snapshots must be selected wisely, depending on the application, and their acquisition will take 
some time, the acquisition interval. The acquisition of the training data and the estimation of the covariance matrix influence the rank.

Other signal properties than the rank which we consider in this article are the DOA (Direction of Arrival) spectrum and eigenpatterns. The DOA spectrum shows the distribution of received power from different DOAs. Eigenpatterns are formed by using the eigenvectors of $\widehat{\mathbf{R}}$ as beamforming weights when plotting the antenna array factor. See Section 3.5 for more details.

1.2. Applications. In interference suppression in radar, the rank of direct and scattered signals is important. Such interference can be direct path jamming (signals from a jammer travelling one-way line-of-sight to the radar), clutter (signals from the radar transmitter travelling to a surface, where they are undesirably scattered back to the radar, also called cold clutter), and hot clutter (signals travelling one-way from a jammer to the radar, not directly but scattered on a surface). The received radar signal in a pulse-Doppler radar can be stored in a radar data cube with dimensions for antenna channels (space), pulses (slow-time), and range bins (fasttime). Suppression of interference is commonly performed with linear filters, which can be one-dimensional, twodimensional, or three-dimensional. For suppression of direct path jamming, space-only snapshots are usually employed, for cold clutter usually space-slow-time and for hot clutter usually space-fast-time.

The output of the suppression filter is $y=\mathbf{w}_{a}^{H} \mathbf{x}$, where $\mathbf{x}$ is a received snapshot. The filter weights are usually computed as [3-5]

$$
\mathbf{w}_{a}=\mu \mathbf{R}_{\mathbf{q q}}{ }^{-1} \mathbf{w}_{0},
$$

where $\mu$ is a scalar and $\mathbf{R}_{\mathbf{q q}}$ is the covariance matrix of the interference plus (receiver) noise signal vector $\mathbf{x}_{\mathbf{q}}$. The vector $\mathbf{w}_{0}$ contains the weights without adaptation. It is usually the steering vector towards the target, possibly with tapering to reduce the sidelobes [5]. The use of filter (2) is commonly called adaptive beamforming for space-only snapshots or STAP (Space-Time Adaptive Processing) for spacetime snapshots (this is also called optimal beamforming and optimal STAP if $\mathbf{R}_{\mathbf{q q}}$ is known and adaptive beamforming and adaptive STAP if $\mathbf{R}_{\mathbf{q q}}$ is estimated). The interference rank determines the needed DoFs (degrees of freedom $=$ number of filter coefficients minus one) of the filter and the needed number of training snapshots (see, e.g., [4]). The DoF should be at least as many as the rank. There are many proposed methods, called reduced rank methods, for the suppression, with the DoFs adapted to the rank; for example, see $[4,6,7]$.

Clutter in bistatic radar (the transmitter and receiver geographically separated) is similar to the case of hot clutter in normal (monostatic) radar. It has been suggested that also bistatic clutter should be suppressed by STAP [8]. Many high resolution DOA, Doppler, and range estimation methods in radar also need to know the signal rank.

In applications other than radar, the signal rank is also needed. In Electronic Support Measures (ESM), a kind of electronic warfare, the objective is to learn as much as possible about noncooperative radar and radio transmitters.
Among other things, it is desired to estimate the number and location of the transmitters. This information can be used as information only or as a help for jamming. Some methods for estimating the number of emitters and their location need the signal rank. In wireless communication it is suggested to use adaptive beamforming and DOA estimation methods for interuser interference suppression and signal separation [1]. For estimating multipath channel models in wireless communication, DOA estimation can be used [9]. For adaptive beamforming and DOA estimation the signal rank is often needed. In MIMO (Multiple Input Multiple Output) communications, the signal rank is directly related to the transmission capacity.

1.3. Description of Work and Outline of Article. This article describes how we have designed and executed low-cost indoor measurements of direct and scattered signals. Direct signals travel one-way in line-of-sight from transmitter antenna to receiver antenna. Scattered signals do not travel in line-of-sight but are scattered on a surface on the way. We have performed the measurements in an anechoic chamber with an experimental array antenna where the received signals arrived directly from the transmitter(s) (direct signals) or were scattered on a moving rough surface reflector (scattered signals). Our experiment takes several aspects into account: transmitted signals with different correlation, decorrelation of the signals during the acquisition interval, covariance matrix estimation, noise eigenvalue spread, calibration, nearfield compensation, and scattering on a rough surface. An advantage of indoor measurements in an anechoic chamber compared to outdoor measurements is the good control of the influencing factors, which is necessary to draw objective conclusions.

The main result of this paper is the design of the experiment for characterization of signal properties of direct and scattered signals. Also our measured signal properties could be seen as results. They agree with theoretic and simulated results in the literature. We have not seen such measured results but they should exist.

In [10], how the space-only rank of direct path signals was dependent on several factors for our experimental array antenna was studied. Also, noise properties were studied. Part of the material in this paper has earlier been published in [11] but the current article contains more details and a significantly deeper analysis.

In Section 2 we will discuss the relation between rank and eigenvalues and also motivate why we can measure spacetime rank with space-only snapshots. Then in Section 3 the experimental setup is described and Section 4 gives some measurement results. A discussion is carried out in Section 5 and, finally, conclusions are presented in Section 6.

\section{Some Preliminaries}

What now follows is a discussion of some topics that are needed for and which motivate the article.

2.1. Rank and Eigenvalues. The rank of a covariance matrix is equal to the number of eigenvalues larger than zero. 


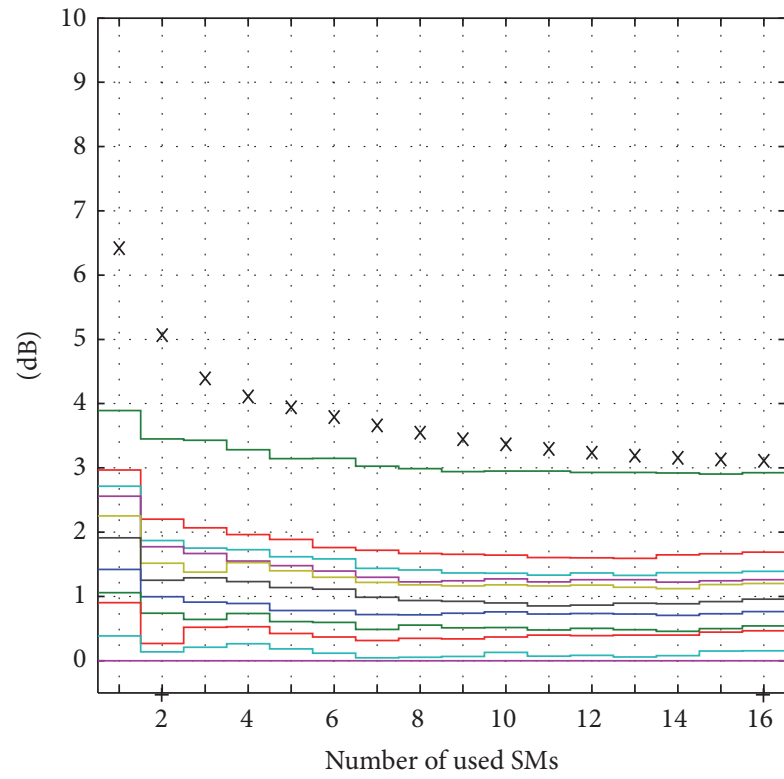

(a)

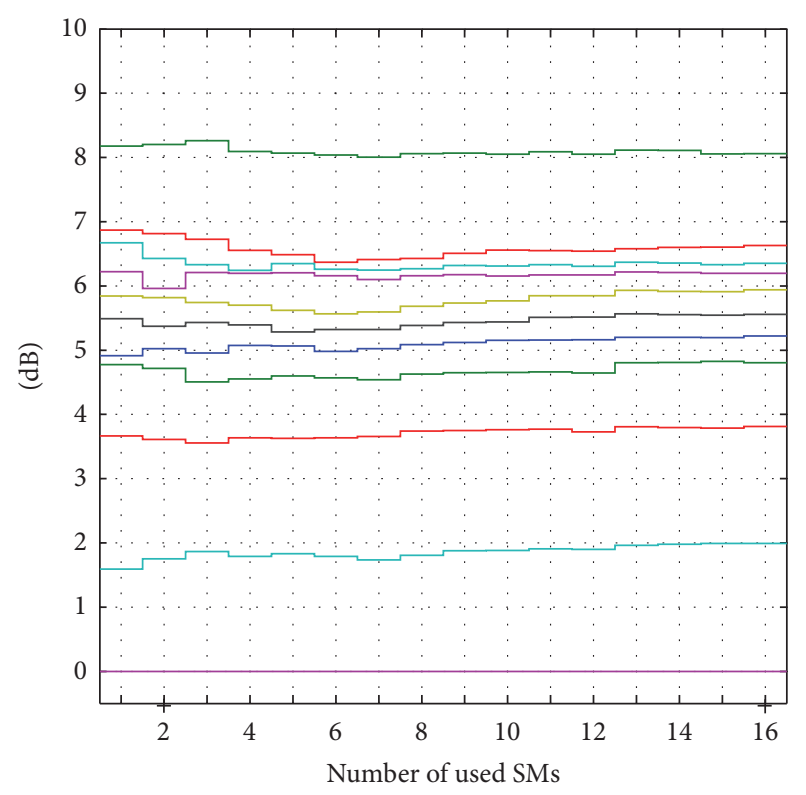

(b)

FIGURE 1: Noise eigenspectrum in measurement C1W (one transmitter and reflector; see Table 1). (b) With spatial calibration using a decoupling matrix and (a) without calibration. dB scale. See Section 3.3 for "SM." See Section 3.5 for an explanation of the figure.

However, what matters for interference suppression [4] and number of sources and DOA estimation [1] is the number of eigenvalues larger than the white noise power. This number is called the effective rank [4]. Theoretically, for a known covariance matrix and for white noise as the only signal, all eigenvalues will be equal and also equal to the noise power. This level is called the noise floor. Thus, the effective rank is the number of eigenvalues larger than the noise floor. These eigenvalues are caused by external signals, like targets, clutter, jammers, or radio transmitters and are called signal or interference eigenvalues. The smaller eigenvalues are caused by the receiver noise and are called noise eigenvalues.

In reality the noise eigenvalues will not be equal. There are two reasons. First, the estimated eigenvalues will be different, even if the true covariance matrix has equal eigenvalues, because of estimation errors $[4,10]$. If these incorrectly estimated noise eigenvalues are used in the optimal filter (2), the performance will be degraded [4]. Two possible solutions are to set the noise eigenvalues to their correct value (by calibration or appropriate estimation [12]) or to use diagonal loading [4]. When setting the correct noise eigenvalue, the number of signals/interference eigenvalues must be known.

The second reason for different noise eigenvalues is that the true eigenvalues really are different due to system nonidealities, like unequal noise power in the channels or correlation between the channels, or due to the used calibration, for example, with a decoupling matrix (Figure 1 and Section 3.2). These true unequal noise eigenvalues should not be made equal since the optimal filter (2) needs the true covariance matrix, including true unequal noise eigenvalues.

To determine the number of signals/interference eigenvalues (of direct and scattered signals) we compute in this article a threshold $\lambda_{T}$ as the maximum eigenvalue of a measured and estimated noise-only covariance matrix, normalized with the minimum eigenvalue. The threshold then includes the effects due to finite number of snapshots and to nonidealities of the system like unequal and correlated channel noise. The eigenvalues below the threshold are caused by the system (noise and nonidealities) and weak signal/interference eigenvalues. The eigenvalues above the threshold will then, hopefully, only be caused by the external signal/interference sources. Eigenvalues above the threshold will be called large eigenvalues.

2.2. Hot Clutter and Space-Only Data. Hot clutter suppression is an important use of our results. Therefore we here explain why our space-only measurements of direct and scattered signals are relevant for hot clutter.

The theoretic results in [14] about the estimated spacefast-time hot clutter covariance matrix indicate that the rank of this matrix can be measured by the space-only covariance matrix, if the number of scatterers seen by the receiver is less than the size of the space-only snapshot (which is the case in our measurements since in all experiments the number of large eigenvalues, $\max 10$, is less than the size of the snapshot, 12; see Table 2). Fast-time effects, like jammer and system bandwidth and time-delay to the scatterers, are included in the theoretic model and affect the space-only rank through varying decorrelation of the signals from different scatterers. What determine the rank of space-only or space-fast-time signals are the scatterers and not the number of used samples in space or fast-time. Note that the results in [14] are valid for the estimated covariance matrix (1). This is the covariance matrix that must be used in the signal processing. This is also 
the one which is used in the analysis of our measurements. With an estimated covariance matrix the acquisition interval, during which decorrelation can occur, is inevitable.

In [15] the authors measure channel rank in indoor wireless communications by the rank of the time-only covariance matrix of the received signal. They say that in narrowband systems the channel rank is equal to the number of resolvable multipaths for uncorrelated scattering, which with our terminology is the number of uncorrelated sources. This confirms that what determine the rank are the scatterers and not the number of used samples in space or time.

\section{Experimental Setup}

3.1. The Experimental Array Antenna. The experimental receiver antenna $[10,16]$ used in this article was designed and built by FOI (the Swedish Defence Research Agency). The high quality antenna has sidelobe levels below $-60 \mathrm{~dB}$ $[10,16]$ and DOA estimation resolution below one-tenth of the conventional beamwidth $[10,17]$. The antenna consists of a horizontal receiving linear array of 12 antenna elements with slightly less than half a wavelength separation $(45 \mathrm{~mm})$, 12 receiver modules, $12 \mathrm{~A} / \mathrm{D}$ converters (12 bits), and 12 buffer memories. The antenna has an agile frequency band of $2.8-3.3 \mathrm{GHz}$ and an instantaneous bandwidth of $5 \mathrm{MHz}$. The antenna elements are vertically polarized and have a horizontal $3 \mathrm{~dB}$ beamwidth of about $115^{\circ}$ and a vertical beamwidth of about $15^{\circ}[10]$. The horizontal beamwidth of the whole array is about $10^{\circ}$. The receiver modules were manufactured by Ericsson Microwave Systems (today Saab Electronic Defence Systems). From the buffer memories the signals are transferred to a standard computer, where the IQconversion, DDC (digital downconversion and downsampling with a factor of 4), calibration correction, and spatial signal processing are performed in nonreal time. See the hardware block diagrams in [16].

The noise properties of our experimental antenna have been investigated by Pettersson in [10]. He stated that the noise sources, without external transmitters, are mainly internal thermal noise from the receiver modules and external thermal noise from the anechoic chamber walls. With external transmitters, there may be additional noise sources, like sampling jitter and phase noise of the signal generators. The true noise power is different in the channels [10] and it may differ by up to $1.5 \mathrm{~dB}$. The noise also has a small correlation between the channels. The absolute value of the nondiagonal elements of the noise covariance matrix can be up to about one-tenth of the diagonal elements [10]. These two noise properties will give spread of the estimated noise eigenvalues; see Section 2.1.

3.2. Calibration. Accurate channel equalization (for frequency response) and spatial channel calibration (for mutual coupling) are utilized $[10,16]$. The spatial calibration can be performed with three different methods [10]

(i) with a DOA correction table on the steering vectors,

(ii) with a decoupling matrix on the steering vectors,

(iii) with a decoupling matrix on the antenna signals $\mathbf{x}$.

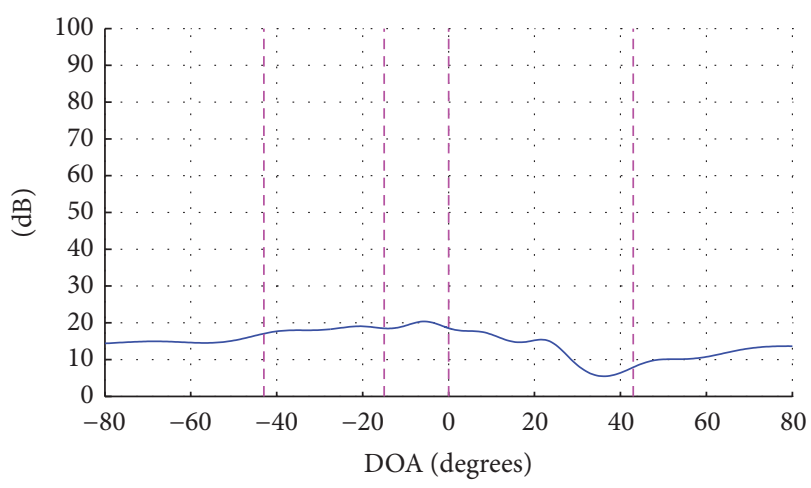

FIGURE 2: Capon DOA spectrum without spatial calibration. Otherwise the same measurement (U2SS, two uncorrelated strong transmitters; see Table 1) and processing as in Figure 10. See Section 3.5 for an explanation of the figure.

The fourth potential method, using a DOA correction table on the antenna signals, is not possible because these signals do not correspond to a single and known DOA. We prefer using a correction table on the steering vectors (method (i)) whenever possible. We have not found any drawbacks with storing such a table instead of only a decoupling matrix, which is contrary to the opinion in [18].

If in STAP the spatial calibration is performed on the antenna signals $\mathbf{x}$ (by method (iii)), then the same calibration should be done on the antenna signals $\mathbf{x}_{n}$ utilized for the estimation of the interference covariance matrix (1). The reason is to keep the STAP filter (2) optimal, for example, keeping the filter as a matched filter. However, if the spatial calibration is applied on the signals, the internal noise will become more correlated, due to the decoupling matrix [10], and the spread of the noise eigenvalues will be increased (Figure 1 and Section 2.1).

Without any spatial calibration the interference suppression performance will be degraded significantly. See Figure 2 for an example with a Capon DOA spectrum (Section 3.5) and compare with Figure 10 where spatial calibration is applied (via a DOA correction table on the steering vectors). See also [18]. The Capon spectrum is a form of the STAP filter (2).

3.3. Reflector and Data Acquisition. The measurements were performed in an anechoic chamber at FOI. Both the array antenna and the reflector were horizontally oriented (Figure 3). The reflector, made of a fine-meshed aluminum net of size $4.0 \mathrm{~m} \times 1.5 \mathrm{~m}$, was irregularly dented. It was designed to simulate a rough surface with a Gaussian height distribution (with a standard deviation somewhat less than one wavelength) and a Gaussian height correlation function (with a correlation distance of some wavelengths). This surface was chosen to obtain a sufficient number of scattering points from hills and valleys and sufficient roughness to have more than a wavelength bistatic range variation due to the surface roughness. We did not aim to model different terrain types but to achieve multipaths and obtain decorrelation by movement. 


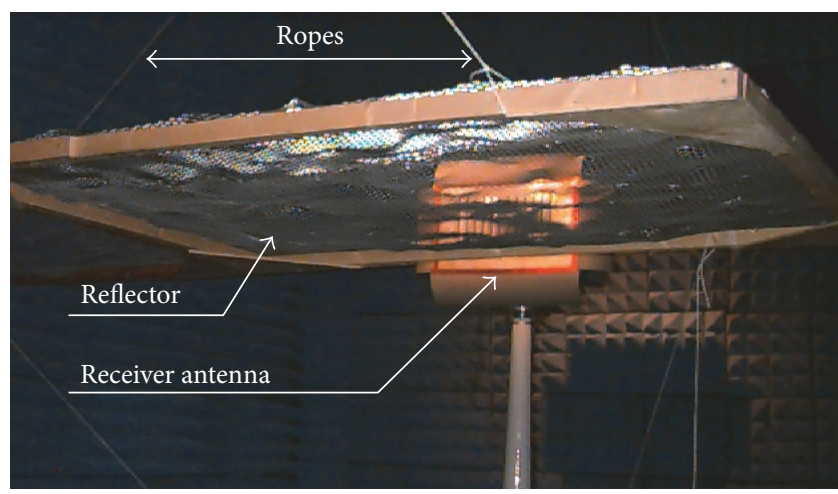

FIGURE 3: The reflector and the receiver antenna in the anechoic chamber. Photo from [13].



Figure 4: Top view of transmitter and receiver antennas and the reflector in the anechoic chamber. The drawing is not to scale.

The reflector was suspended from the ceiling using thin ropes at a height which gave grazing angles of about $9^{\circ}$. This grazing angle was just outside of the $3 \mathrm{~dB}$ elevation beamwidth $\left(7.5^{\circ}\right)$ of the experimental antenna. This geometry was chosen to have an unobstructed view of the antenna for the direct path signal and to have a sufficient delay corresponding to about one wavelength to obtain a large phase shift for the scattered signals. See Figures 4 and 5 for placement of the equipment in the chamber. The suspension allowed the reflector to swing easily from one side to the other.

When the reflector was swinging back and forth, with a deviation of one to two wavelengths, several submeasurements (SMs) were conducted with a delay of $15 \mathrm{~s}$ between the SMs. Each SM contained 256 snapshots (after downconversion and downsampling) and took $40 \mu$ s to measure. These snapshots were utilized to estimate a covariance matrix (1). The used covariance matrix in the analysis (Section 3.5) is the average of the covariance matrices from the used SMs. The total time for all SMs was about $3 \mathrm{~min}$ for 12 SMs (3072 snapshots), $4 \mathrm{~min}$ for $16 \mathrm{SMs}$ (4096 snapshots), and $6 \mathrm{~min}$ for 24 SMs (6144 snapshots). Increasing the number of used SMs in this study corresponds to increasing the acquisition interval in [14, 19] (denoted as $T$ in [19]). An acquisition interval is needed to estimate the covariance matrix (1).

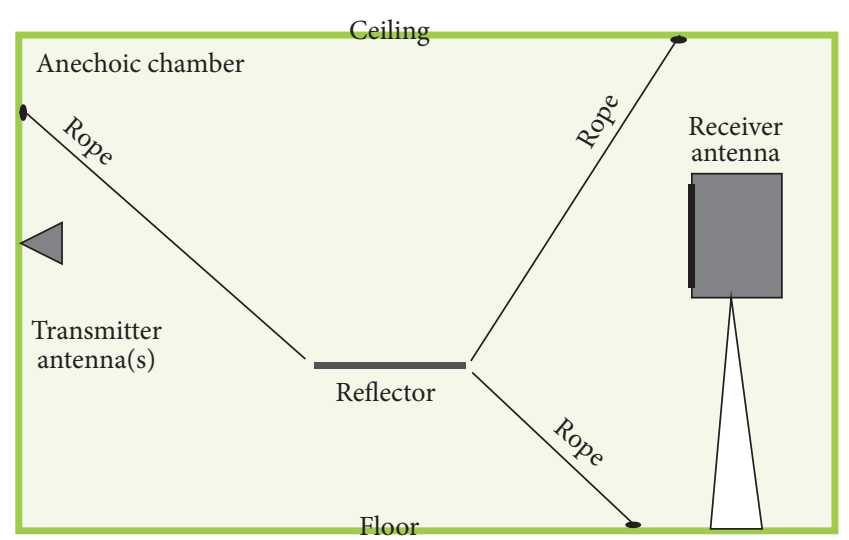

FIGURE 5: Side view of transmitter and receiver antennas and the reflector in the anechoic chamber. The drawing is not to scale.

By utilizing several SMs and a swinging reflector we could simulate decorrelation of the direct and scattered signals. The movement of the reflector gave a random component in the phase of the signal. By this the different multipath signals decorrelated with each other and with the direct signal. The movement of the reflector also enabled us to measure an "average" reflector instead of a particular one by using the same reflector at different positions.

The use of the reflector was not meant to replicate the exact generation of cold or hot clutter or any other signal/ interference. In applications the decorrelation can occur due to movement of transmitter and receiver, nonzero bandwidth, and so forth (see above and Section 5).

3.4. Transmitters. We used one or two transmitter antennas, which were positioned at about the same height as the receiver antenna. The transmitter antenna 1 was located at the broadside of the receiver antenna and antenna 2 was shifted in DOA (Direction of Arrival) by $15^{\circ}$, which is 1.5 beamwidths of the receiver antenna; see Figure 4. Transmitter antenna 1 was a rectangular standard gain horn with a horizontal $3 \mathrm{~dB}$ beamwidth of $30^{\circ}$. The second transmitter antenna was a conical ridge horn. The receiver antenna was directed towards transmitter antenna 1 , which had DOA $0^{\circ}$ seen from the receiver antenna array center.

The distance between the transmitter antenna 1 and the receiver antennas was $6.0 \mathrm{~m}$, which is on the limit to be considered a far-field distance for one antenna element. Near-field corrections in the receiver antenna were therefore applied $[10,16]$. The far-field (Fraunhofer) region for the

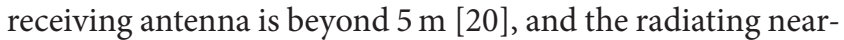
field (Fresnel) region is between 0.7 and $5 \mathrm{~m}$. This means that the reflector is in the Fresnel region with almost three times the distance from the reactive near-field. We can therefore assume that there is no coupling between the antenna and the reflector and that the reflector will not influence the receiving antenna properties. By this we conclude that the antenna setup will not influence the decorrelation properties investigated in the article.

One or two commercially available signal generators were used for the transmitters. The transmitted waveforms were 


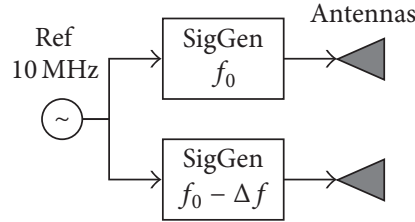

Uncorrelated transmitters

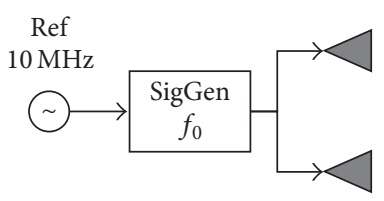

Coherent transmitters

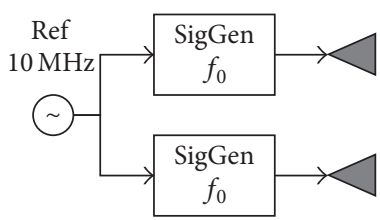

Intermediate correlated transmitters
FIGURE 6: Generation of uncorrelated, intermediate correlated, and coherent transmitters. An integral number of periods of the uncorrelated signals fit exactly in the acquisition interval. The frequency $f_{0}$ was about $3 \mathrm{GHz}$ and $\Delta f$ about $0.4 \mathrm{MHz}$.

pure sinusoids, that is, the carrier signal without modulation. Measurements were conducted with coherent, intermediate correlated, and uncorrelated transmitted signals (Figure 6). The coherent signals originated from the same signal generator which fed both antennas. The uncorrelated signals were generated by two signal generators with different frequencies. The frequencies were selected so that integer numbers of periods of the sinusoid signal of each transmitter (after downconversion and downsampling) were received during the data acquisition interval. The two transmitted signals then seemed uncorrelated over this interval. This matter is treated in [10]. We believe that the accuracy of the frequencies of the transmitters and the internal oscillators of the receivers was sufficiently good to give sufficiently low correlation between the signals which should be "uncorrelated." Completely uncorrelated signals are not necessary. Even if the signals are somewhat correlated, the same qualitative behavior is achieved regarding the eigenanalysis [21]. A similar condition for space uncorrelation was noted in [19]. The intermediate correlated signals were generated by two signal generators with the same frequency. The two signal generators were in all cases phase-locked with a $10 \mathrm{MHz}$ signal. The intermediate correlated signals should therefore be close to coherent. The used frequencies for the transmitters were $2999.596875 \mathrm{MHz}$ and $2999.193750 \mathrm{MHz}$. These frequencies fulfill the requirements described above.

Table 1 lists important measurement parameters of our measurements. The two transmitters were selected to be either nearly equal in strength or very different in strength. This later case could imitate a situation with a weak target signal and a strong jammer. The difference in power, $45 \mathrm{~dB}$, was chosen so that the power of the weak transmitter would be similar to the power of the reflections from the stronger transmitter.

3.5. Methods of Reflection Analysis. Our first analysis type employed to describe the direct and scattered signals is the
TABLE 1: Parameters of the measurements.

\begin{tabular}{lccccc}
\hline Name & $\begin{array}{c}\text { Number of } \\
\text { transmitters }\end{array}$ & Correlation & $\begin{array}{c}\mathrm{PG}^{\mathrm{a}} \\
\mathrm{Tx} \mathrm{1}^{\mathrm{b}} \\
{[\mathrm{dB}]}\end{array}$ & $\begin{array}{c}\mathrm{PG}^{\mathrm{a}} \\
\mathrm{Tx}^{\mathrm{b}} \\
{[\mathrm{dB}]}\end{array}$ & $\begin{array}{c}\mathrm{SNR}^{\mathrm{c}} \\
{[\mathrm{dB}]}\end{array}$ \\
\hline C1S & 1 & - & 0.5 & - & 41 \\
C2SS & 2 & Coherent & 0.5 & -4.2 & 45 \\
U2SS & 2 & Uncorrelated & 0.5 & -4.5 & 40,32 \\
I2SS & 2 & Intermediate & High & High & 42 \\
C1W & 1 & - & -39.5 & - & 14 \\
C2SW & 2 & Coherent & 0.5 & -44.2 & 43 \\
U2SW & 2 & Uncorrelated & 0.5 & -44.5 & $44,-3$ \\
\hline
\end{tabular}

${ }^{a} \mathrm{PG}$ is the effective isotropic radiated power $[\mathrm{dBm}]$.

${ }^{\mathrm{b}}$ The DOA was $0^{\circ}$ for transmitter 1 (Tx 1$)$ and $-15^{\circ}$ for transmitter 2 (Tx 2 ).

${ }^{c}$ The SNR is for one antenna channel (mean value between the channels) and one time sample after IQ, DDC, and calibration and is estimated from data (by the frequency spectrum, not the Capon spectrum). For measurements C2SS, I2SS, and C2SW the stated SNR is for the sum of the two transmitters. The reason for this is that the transmitters could not be separated in the SNR estimation.

TABLE 2: Summary of eigenspectra results ${ }^{\mathrm{a}}$.

\begin{tabular}{lccccc}
\hline Name & Figure & 1SM & Increase per SM & 12 SMs & 24 SMs \\
\hline C1S & Figure 8 & 1 & 1 & 8 & 8 \\
C2SS & Figure 9 & 1 & $\leq 1$ & 7 & 8 \\
U2SS & Figure 10 & 2 & $\begin{array}{c}\text { 2 (up to 6 SM), } \\
\leq \text { (above 6 SM) }\end{array}$ & 10 & 10 \\
I2SS & Figure 11 & 2 & $\leq 1$ & 9 & 10 \\
C1W & Figure 12 & 1 & 0 & 1 & 1 \\
C2SW & Figure 13 & 1 & $\leq 1$ & 8 & 8 \\
U2SW & Figure 14 & 2 & 1 & 8 & 8 \\
\hline
\end{tabular}

${ }^{\mathrm{a}}$ The table gives the number of large eigenvalues, that is, eigenvalues larger than the threshold $\lambda_{T}$. Often the last large eigenvalue came later than the rest.

${ }^{\mathrm{b}}$ Approximate values.

Capon DOA spectrum [22] (also called MVDR, Minimum Variance Distortionless Ratio),

$$
P_{\text {capon }}(\theta)=\frac{1}{\mathbf{a}^{H}(\theta) \widehat{\mathbf{R}}^{-1} \mathbf{a}(\theta)},
$$

where $\mathbf{a}(\theta)$ is the spatial steering vector and $\widehat{\mathbf{R}}$ is the estimated covariance matrix in (1). The steering vector is a model of how the receiver perceives an impinging signal from direction $\theta$. For us $\mathbf{a}(\theta)$ was measured in the anechoic chamber and tabulated for $-80^{\circ} \leq \theta \leq 80^{\circ}$ with a step of $0.5^{\circ}$. For angles $\theta$ between the ones in the table the vector $\mathbf{a}(\theta)$ was interpolated linearly. The calibration correction for antenna element coupling, amplitude and phase drift, and near-field were all done on the steering vectors. This is method (i) in Section 3.2 (DOA correction table on the steering vectors). See $[10,16]$ for more information.

The Capon spectrum shows the distribution of received power from different DOAs unless the signals are coherent. The Capon spectrum also gives an indication of how well optimal beamforming and STAP can suppress scattered signals, since it is computed according to (2), with special 


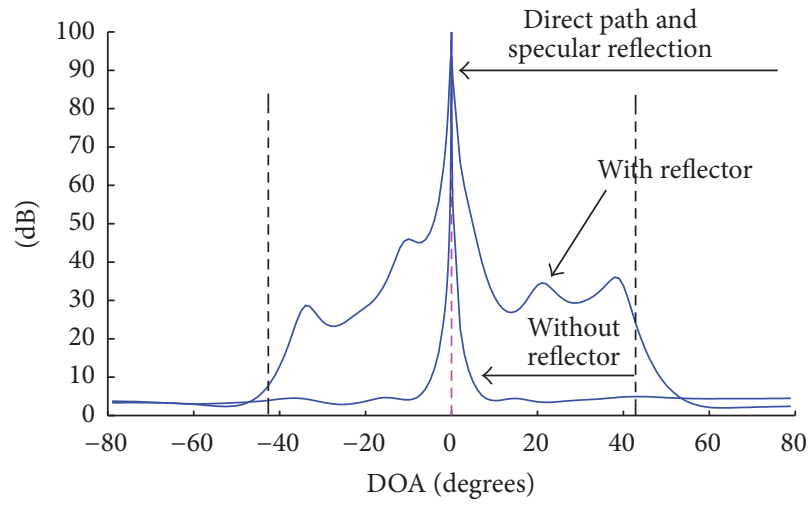

FIGURE 7: Capon DOA spectra with the reflector (measurement C1S in Table 1, same result as Figure 8) and without the reflector plotted on top of each other. The region between the left and right vertical dashed lines is the reflection region, that is, where reflections are possible because of the presence of the reflector. The middle dashed line(s) is the true DOA of the direct signal(s). 24 SMs.

choices of the covariance matrix and the scalar $\mu$. For the Capon spectra, $24 \mathrm{SMs}$ were used. As an example of the influence of the reflector, Figure 7 shows the Capon spectra for experiments with and without the reflector.

The second analysis type is the eigenspectrum, which is the eigenvalues of the antenna signal covariance matrix, usually sorted in decreasing order. We have plotted the eigenvalues in an uncommon manner. They are not plotted in decreasing order for a single covariance matrix but all eigenvalues for the same covariance matrix are plotted in the same "column." The different columns are used for covariance matrices with different number of SMs. We have computed the eigenvalues for an increasing number of used SMs, up to a maximum of 24. However, in the graphs in this article only up to 16 SMs are plotted, due to space limitations. See Figure 8(b) for an example. The eigenspectrum illustrates the signal/interference rank. In each presentation we have normalized the eigenvalues to the smallest one. No spatial calibration was performed for the eigenspectra results (except as an illustration in Figure 1). For interference suppression this is not necessary, since when computing the optimal weights in (2), the calibration can and should be performed on the quiescent weight vector $\mathbf{w}_{0}$ (method (i) in Section 3.2 ) instead of the covariance matrix (via a decoupling matrix on the received signals used to estimate the covariance matrix, method (iii)). Done differently, the noise eigenvalue spread would increase (Figure 1) and the noise eigenvectors would influence the optimal filter (2) more and perhaps require more DoFs. In this paper we determine the interference rank by the threshold described in Section 2.1. In the graphs the threshold is marked by the symbol " $x$ "; see Figure 8 .

We also present eigenpatterns (eigenvector antenna array factors) [23]. Eigenpatterns are formed by using the eigenvectors of the antenna signal covariance matrix as beamforming weights when plotting the antenna array factor. Since the element pattern is not included in the steering vector, our eigenpatterns will not be antenna patterns. For the eigenpatterns the spatial calibration was performed using a decoupling matrix on the training signals (method (iii) in Section 3.2). See $[10,16]$ for more information. Since the reflector is not placed in the extreme near-field and the eigenpatterns are transformed to the far-field (by near-field compensation on the training signals and by the used far-field steering vector), there should be no significant differences in the eigenpatterns compared to the case where the reflector is in the far-field.

\section{Measurement Results}

4.1. Capon DOA Spectra and Eigenspectra. The Capon DOA spectrum and the eigenspectrum for measurement C1S (a single, strong transmitter; see Table 1) are shown in Figure 8. A clear peak at DOA $0^{\circ}$ is seen in Figure 8(a). This is the direction of the transmitter. The peak contains both the direct signal and the specular reflection. In the figure the extension of the reflector is given by dashed vertical lines. As seen in the figure, most reflections from the reflector are about $60 \mathrm{~dB}$ lower than the peak. We see that the whole reflector is covered by the power from the transmitting antennas. In Figure 8 (b), the number of large eigenvalues, that is, above the threshold (Section 2.1), starts with one and increases by one for each new SM, except for the last large eigenvalue, up to a maximum of eight. This means that we can consider the signal/interference rank to be about one to eight, depending on the level of decorrelation, of which the direct signal is one. Table 2 summarizes all eigenspectra.

Figure 9 presents results from measurement C2SS (two strong coherent transmitters). Here, the Capon spectrum peak for DOA $0^{\circ}$ is considerably lower, $30 \mathrm{~dB}$, than in Figure 8 . The second direct signal peak is also weak and has some bias in DOA. Most parts of the reflection region are weaker than in Figure 8. The probable reason for the low levels is the mutual cancellation of the signals from the two transmitters due to the coherence between them $[21,24]$. The eigenspectrum is similar to Figure 8 . This similarity means that two coherent transmitters are seen as a single transmitter. The largest eigenvalue in Figure 9 has nearly the same size as in Figure 8, despite the largest peak being lower in Figure 9 than in Figure 8. This is possible since the eigenvalues do not correspond directly to the power of the signal sources but the 


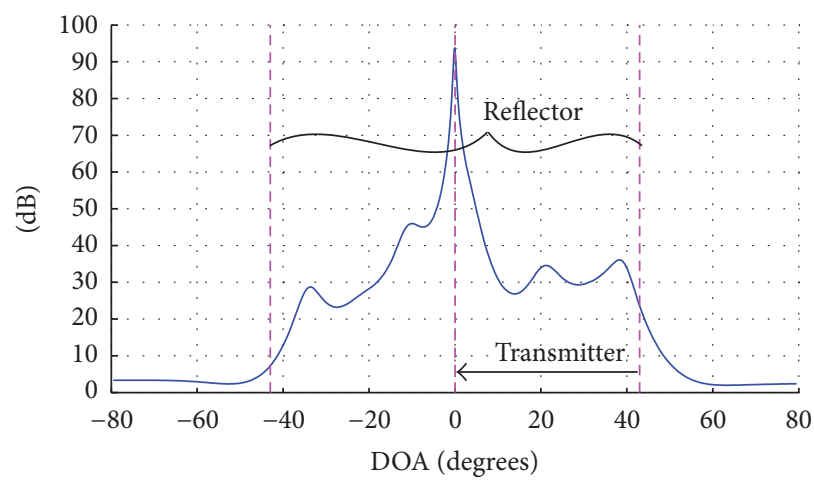

(a)
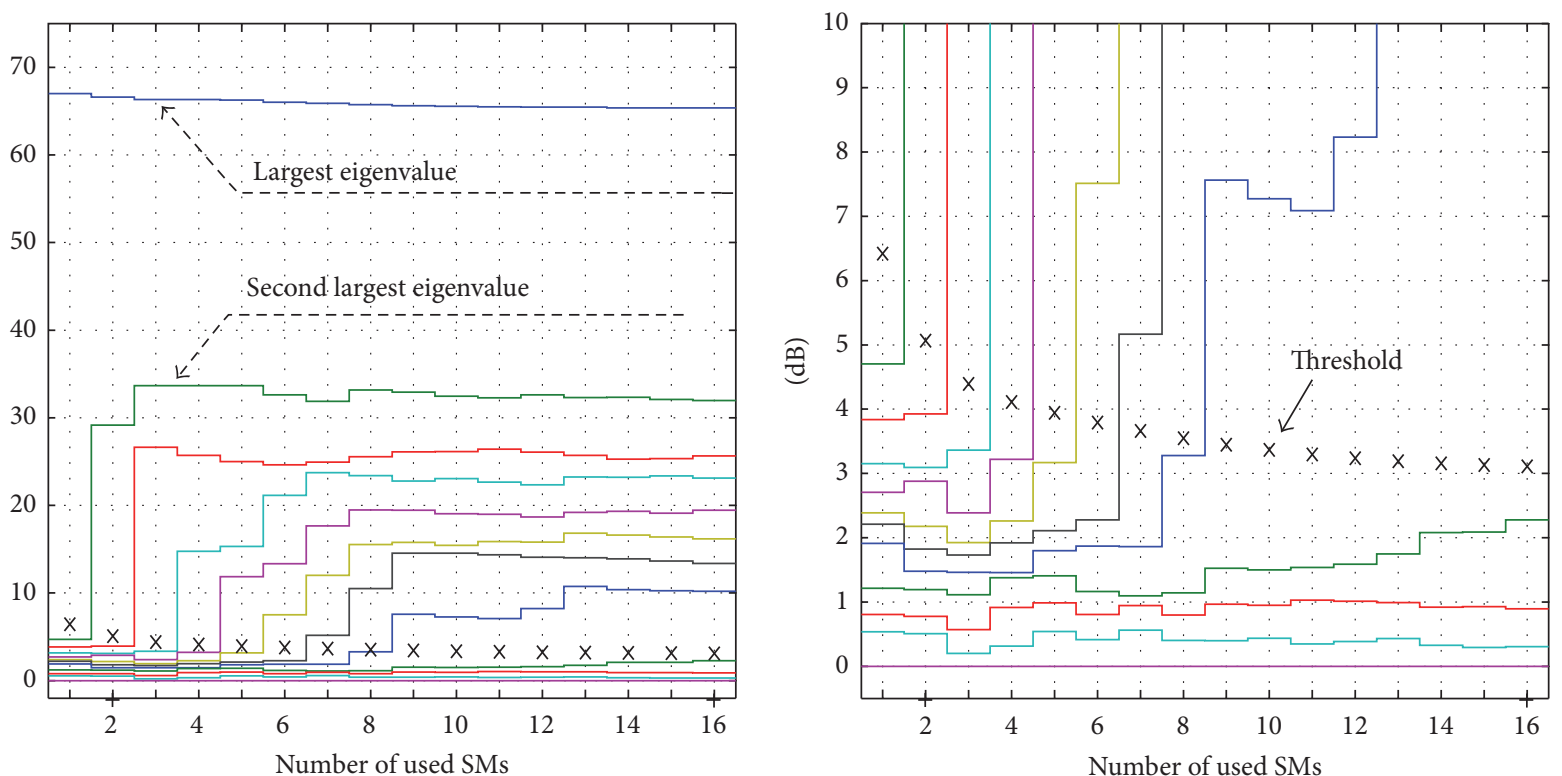

(b)

Figure 8: Measurement C1S. A single strong transmitter. (a) Capon DOA spectrum. Figure description in Figure 7. (b) Eigenspectrum. Zoom-in to the right. dB scale. See Section 3.3 for "SM."

sum of the signal eigenvalues $\lambda_{l}$ is equal to the sum of the signal powers $P_{l}[21]$ :

$$
\sum_{l=1}^{L} P_{l}=\sum_{l=1}^{L} \lambda_{l}
$$

where $L$ is the number of signal sources.

The case with two strong uncorrelated transmitters (measurement U2SS) is depicted in Figure 10. Both direct signals (including specular reflections) are clearly seen. Also the reflection region is clearly visible. When studying the eigenspectrum, we note a difference to the previous measurements. Here it starts with two large eigenvalues for the first SMs and initially increases by two for each new SM (up to six). Then it increases slower, probably because some eigenvalues are below the noise floor, up to ten large eigenvalues on the remaining SMs.
Figure 11 shows Capon spectrum and eigenspectrum for the case with two strong and intermediate correlated transmitters (measurement I2SS). The Capon spectrum seems to be nearly identical with the case of uncorrelated transmitters (compare Figures 10 and 11). The eigenspectrum starts with two large eigenvalues and then increases by only one for each extra SM, except for no change between 3 and 4 SMs. The maximum number of large eigenvalues is ten as for uncorrelated transmitters (U2SS) but the final large eigenvalues require more SMs and therefore more decorrelation than for uncorrelated transmitters. The more uncorrelated the transmitters are, the more equal in size the eigenvalues are in the simulations in $[19,21]$. In measurement I2SS the transmitters were more correlated than in U2SS. The eigenvalues were probably therefore more unequal and some were too small to cross the threshold and become "large" ones. Thus, the signal/interference rank increases as the correlation between the transmitters decreases. 


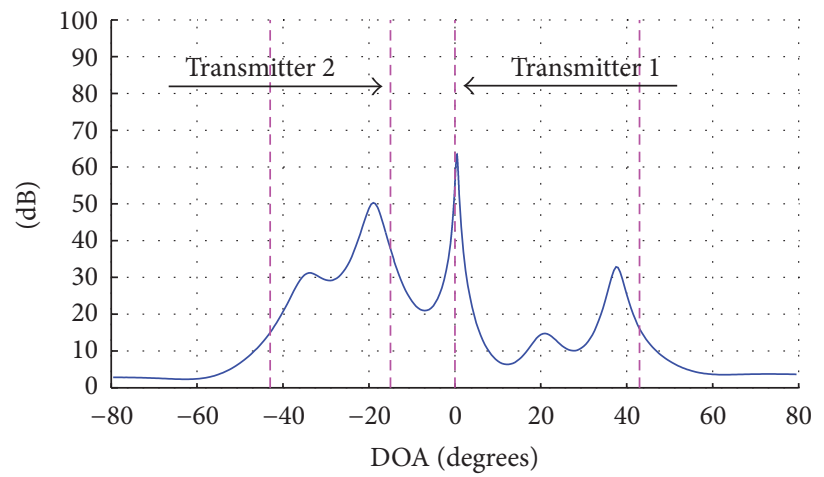

(a)
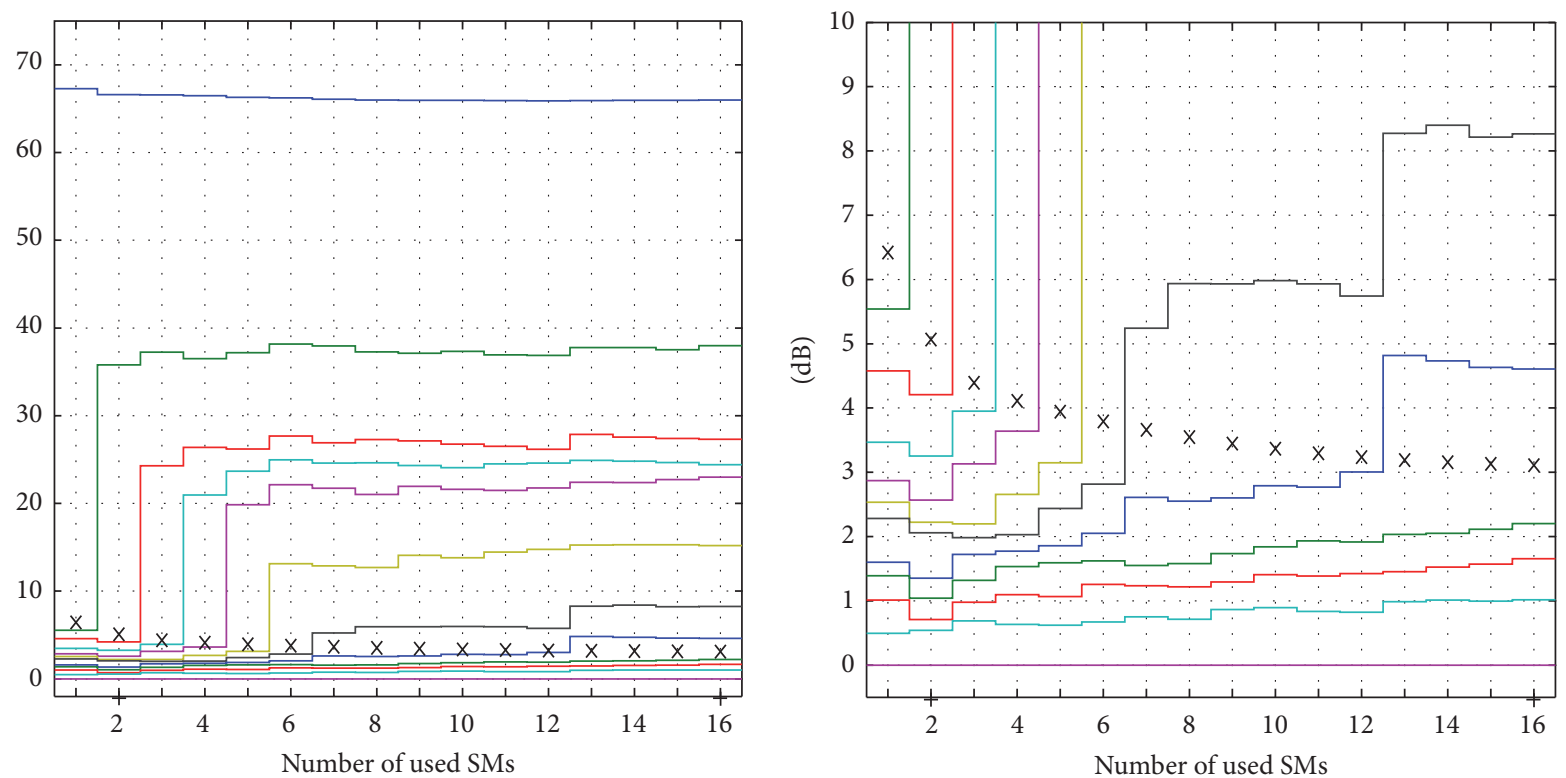

(b)

FIGURE 9: Measurement C2SS. Two coherent strong transmitters. (a) Capon DOA spectrum. (b) Eigenspectrum. Zoom-in to the right. dB scale. See Section 3.3 for "SM."

In Figure 11, we observed that there are two large eigenvalues for a single SM. By changing the analysis to use only 1 SM in 16 repetitions, we obtained two large eigenvalues in 13 repetitions, we obtained one large eigenvalue in 1 repetition, and we obtained three large eigenvalues in 2 repetitions. This shows that with a high probability there will be two large eigenvalues for $1 \mathrm{SM}$.

We now turn to the case with a single weak transmitter (measurement C1W). The Capon spectrum (Figure 12) tells us that the peak is $40 \mathrm{~dB}$ lower than in Figure 8, which is as expected since the transmitted power was this much lower. The reflection region is not seen at all. The explanation is that the scattered signals are weaker than the noise. The eigenspectrum in Figure 12 contains the same information. It has only one large eigenvalue for all SMs, because of the weak transmitter. All but one eigenvalue are below the noise. See also the discussion about the iceberg effect in Section 5.

The Capon DOA spectrum in Figure 13 for two coherent and different strong transmitters (measurement C2SW) resembles the one for a single strong transmitter (Figure 8) very much. Also the eigenspectra (Figure 13(b)) are fairly similar for few SMs (compare Figures 8(b) and 13(b)). For up to $6 \mathrm{SMs}$, the number of large eigenvalues increases by one for each SM as in Figure 8 but the 7th large eigenvalue does not show up until SM 11 for C2SW. The probable reason for the similarity is that the weak transmitter is too weak to disturb the strong transmitter.

In measurement U2SW (two uncorrelated transmitters with different strength) the Capon DOA spectrum (Figure 14) is also rather similar to the one with a single strong transmitter (Figure 8). The direct signal is about $3 \mathrm{~dB}$ lower and the valleys of the reflection region are deeper. Interestingly, the eigenspectrum (Figure 14) starts with two large eigenvalues, which indicates two noncoherent transmitters despite the low power of the weak transmitter, below the noise (SNR $\approx$ $-3 \mathrm{~dB}$ ). This is also possible because of (4). Then the number of large eigenvalues increases by one for each additional SM, which could indicate a single transmitter. It ends with eight 


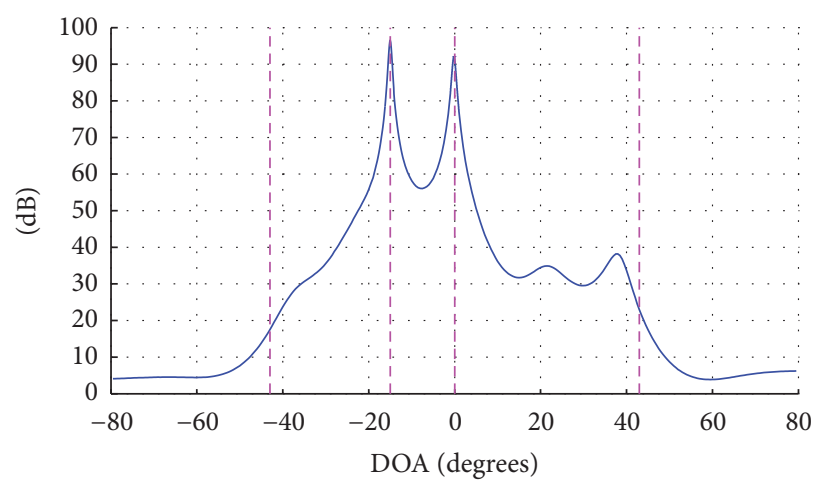

(a)


(b)

FIGURE 10: Measurement U2SS. Two uncorrelated strong transmitters. (a) Capon DOA spectrum. (b) Eigenspectrum. Zoom-in to the right. dB scale. See Section 3.3 for "SM."

large eigenvalues as for a single strong transmitter. There are probably several signal/interference eigenvalues below the noise.

4.2. Eigenpatterns. Figures 15 and 16 give some examples of eigenpatterns. More eigenpatterns can be found in [13] according to Table 3 . We notice that each eigenpattern has one or more large lobes. When the peak of the highest lobe is within the reflection region (where the reflector can give scattered signals, denoted with the outermost dashed vertical lines), we say that the eigenpattern covers the reflection region. When the peak is outside this region we say that the eigenpattern covers the region outside.

In our measurements the eigenpatterns corresponding to the largest eigenvalues usually cover the reflection region. The remaining eigenpatterns cover the region outside. The one or two largest eigenvalues have eigenpatterns which are directed towards the strong direct signals and the other eigenpatterns usually have nulls in these directions (Figures 15 and 16).
Actually, the eigenpatterns, associated with the eigenvectors, must be as "different" as possible since they are orthogonal.

There is approximately the same number of covering eigenpatterns for 1 SM ("without order") as for 12 SMs; see Table 3.

Strangely enough, there is about the same number of eigenpatterns covering the reflection region for $1 \mathrm{SM}$ as there are large eigenvalues using all (24) SMs, especially "without order" (compare Tables 2 and 3). Exceptions are the measurement $\mathrm{ClW}$ which has 7 covering eigenpatterns (Figure 16) despite only 1 large eigenvalue and U2SS and I2SS, which have somewhat fewer covering eigenpatterns than eigenvalues. $\mathrm{C} 1 \mathrm{~W}$ seems to observe all distinct sources with its eigenpattern but not with its eigenvalues. Remember some signal eigenvalues are below the noise floor in the eigenspectra.

For the weak direct signal in the presence of a strong direct signal, its eigenpattern has a bias in DOA. Also for the case with two equal strong coherent transmitters there 


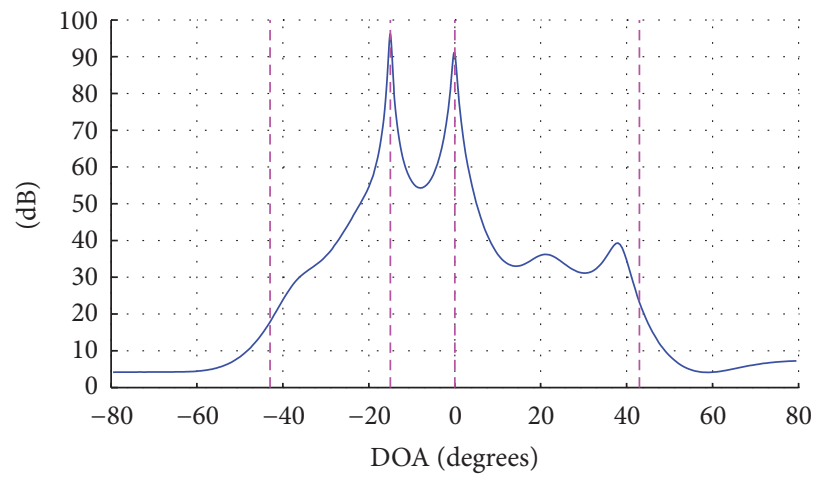

(a)


(b)

FiguRE 11: Measurement I2SS. Two intermediate correlated strong transmitters. (a) Capon DOA spectrum. (b) Eigenspectrum. Zoom-in to the right. dB scale. See Section 3.3 for "SM."

TABLE 3: Summary of eigenpattern results ${ }^{\mathrm{a}}$.

\begin{tabular}{lccccc}
\hline Name & $\begin{array}{c}\text { 1 SM } \\
\text { w ord }^{\text {b }}\end{array}$ & $\begin{array}{c}\text { 1 SM } \\
\text { w/o ord }\end{array}$ & Figure & 12 SMs & Figure \\
\hline C1S & 8 & 8 & 6.8 in [13] & 8 & 6.9 in [13] \\
C2SS & 8 & 8 & Figure 15 & $7-8$ & 6.33 in [13] \\
U2SS & 5 & 7 & 6.24 in [13] & 8 & 6.25 in [13] \\
I2SS & 2 & 6 & 6.36 in [13] & 8 & 6.37 in [13] \\
C1W & 7 & 7 & Figure 16 & $7-8$ & 6.21 in [13] \\
C2SW & 5 & 7 & 6.16 in [13] & 8 & 6.17 in [13] \\
U2SW & 8 & 8 & 6.28 in [13] & 8 & 6.29 in [13] \\
\hline
\end{tabular}

${ }^{\mathrm{a}}$ The table gives the number of eigenpatterns covering the reflection region.

b" $w$ ord" stands for "with order" and means the number of covering eigenpatterns in an uninterrupted sequence from the first one.

" w/o ord" means "without order" and means the total number of covering eigenpatterns.

is a bias, although less than for two signals with unequal strength.
4.3. Summary of the Measurement Results. We have from the measurements obtained results on the rank and other properties of direct and scattered signals. We see that the signal/interference rank depends on the number of transmitters, the SNR (Signal to Noise Ratio), the correlation between the transmitters, and the degree of decorrelation of the transmitter signals that occurs during the data acquisition.

Without decorrelation, the direct and scattered signals of a transmitter will all be coherent. If the scattered signals decorrelate with each other and with the direct signal, the rank is increased. Two coherent transmitters appear as a single transmitter regarding the signal/interference rank. Two strong uncorrelated transmitters give rise to the double number of sources compared to a single transmitter.

With higher SNR, more eigenvalues of the eigenspectrum tail will appear above the noise level, and the rank will be higher.

The eigenpatterns show the reflection region and the DOAs to the direct signals. The eigenpattern can tell us the number of signal sources when the signals still are coherent. 


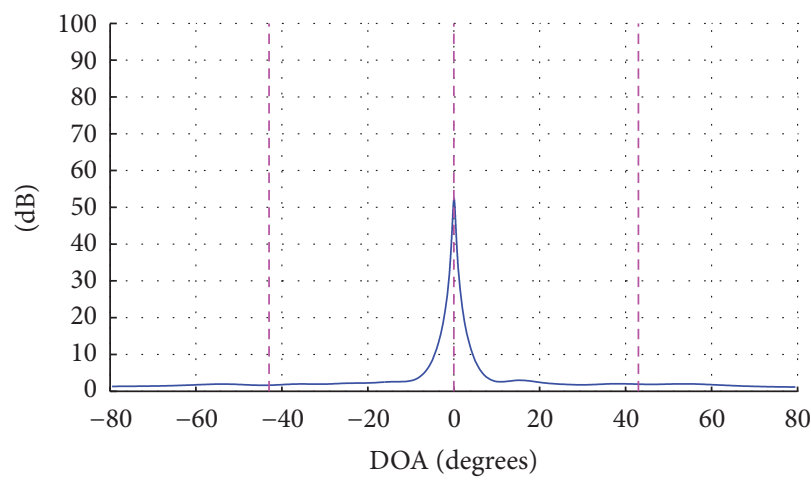

(a)
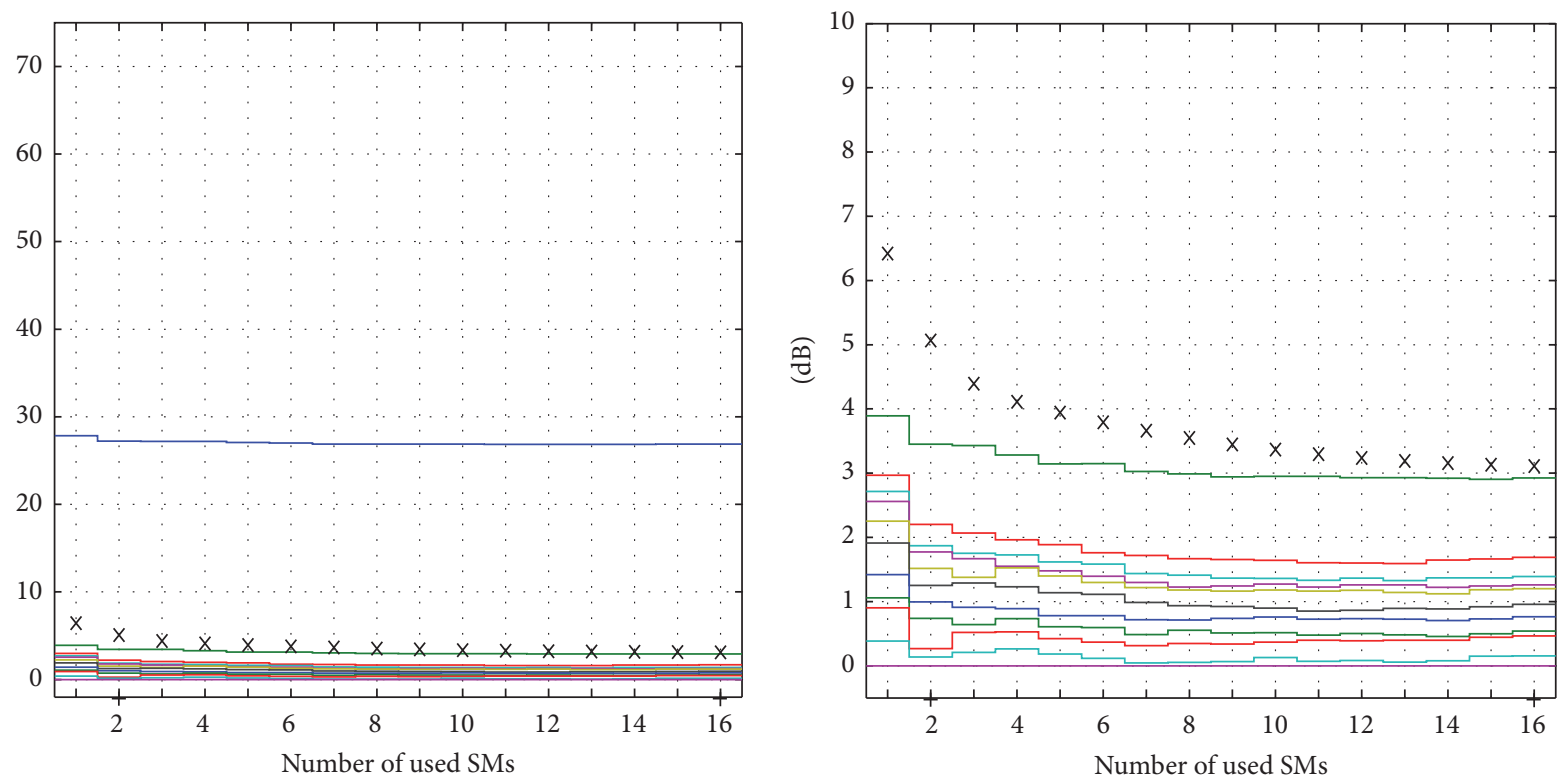

(b)

Figure 12: Measurement C1W. A single weak transmitter. (a) Capon DOA spectrum. (b) Eigenspectrum. Zoom-in to the right. dB scale. See Section 3.3 for "SM."

Alternatively, they can tell us the extent of the reflection region if the number of signal sources is known.

\section{Discussion}

5.1. Discussion about the Experimental System. To show the eigenvalues for an increasing number of used SMs when the reflector is moving gives the possibility to study the signal/interference rank for different degrees of decorrelation. In a real case, decorrelation can occur as a result of platform motion (comparing with [19]), internal clutter motion, nonzero bandwidth [25], long acquisition interval for estimating the covariance matrix, carrier frequency changes, and so forth.

Note that we are studying the estimated covariance matrix (1), not the true covariance matrix. It is the estimated matrix which must be used in algorithms. The measured signal snapshots were space-only snapshots. However, the time dimension enters via the acquisition interval, during which decorrelation of the signals can occur (see also Sections 2.2 and 3.3). This will increase the rank. The decorrelation increases if the acquisition interval is prolonged (more SMs) as in [14].

The Capon spectrum gives a good picture of the impinging power from the direct and scattered signals if the transmitters are noncoherent.

The measurement result will be influenced by different transmitter signals. With a different frequency of the pure sinusoid, there will be different differences in amplitude and phase between the scatterers on the reflector. If the frequency is changed much, the number of scatters will change and thereby the signal rank will change too, with a higher number and higher rank with a higher frequency. Now the signal bandwidth is low and the scatterers on the reflector cannot be resolved in range (= fast-time). A bandwidth in the order of $100 \mathrm{MHz}$ would be needed to resolve in range. If the 


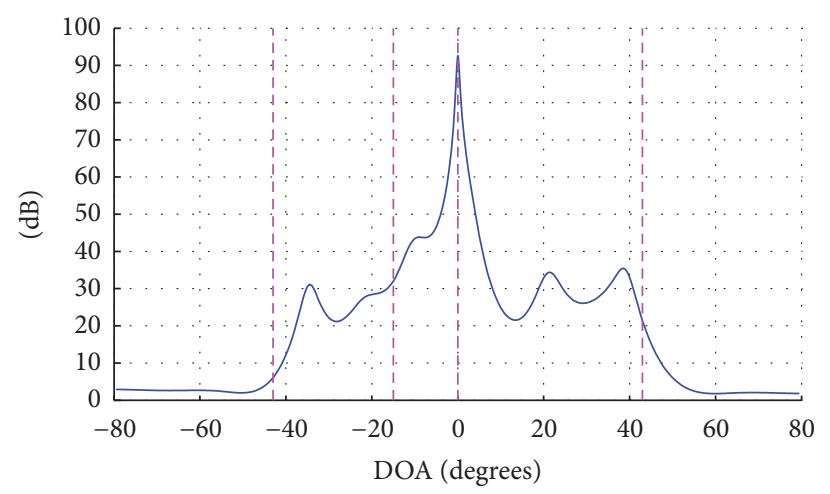

(a)
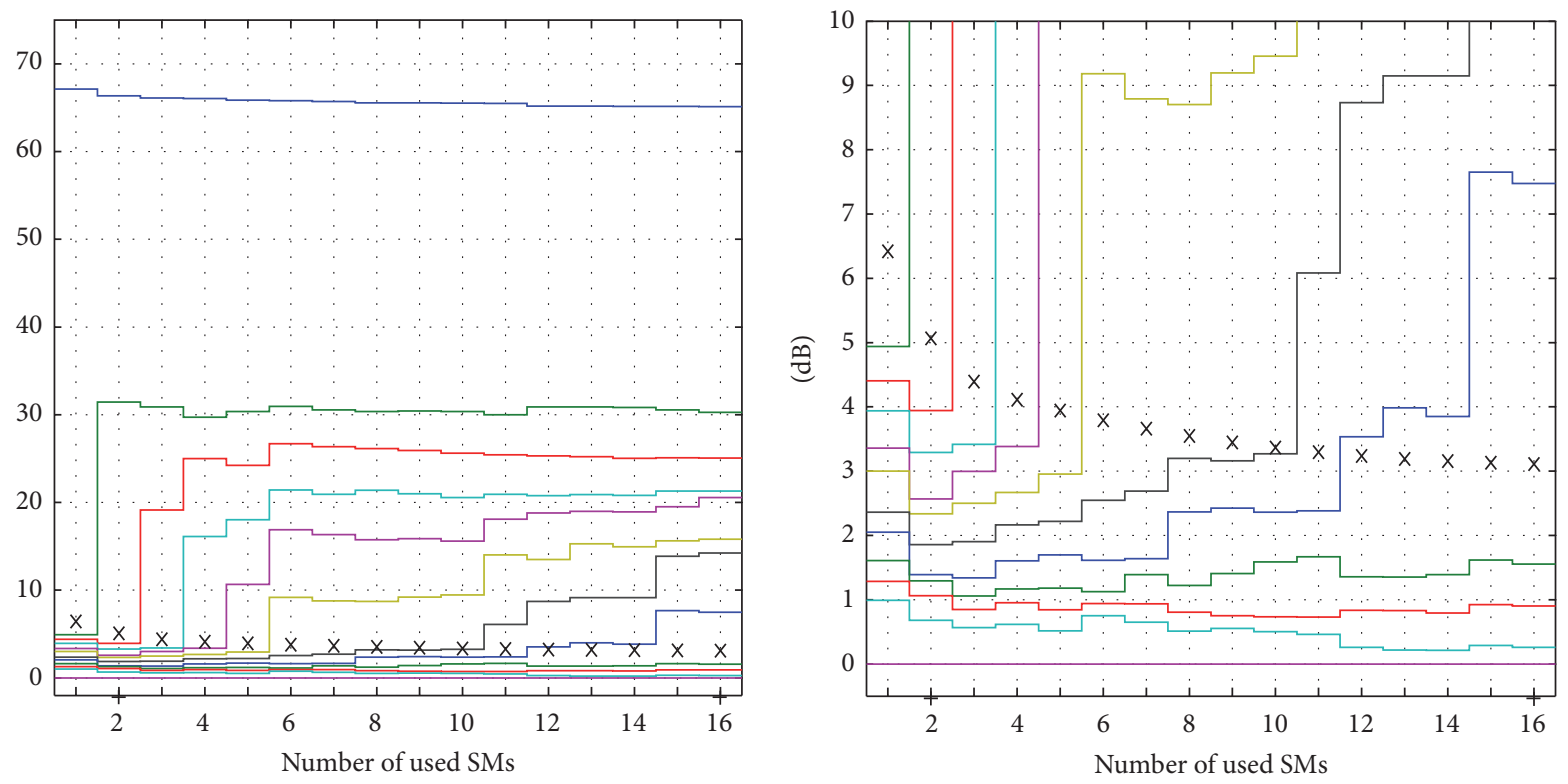

(b)

Figure 13: Measurement C2SW. Two coherent transmitters, one strong and one weak. (a) Capon DOA spectrum. (b) Eigenspectrum. Zoomin to the right. $\mathrm{dB}$ scale. See Section 3.3 for "SM."

transmitter signals are different from pure sinusoids, the emulation of uncorrelated transmitted signals probably had to be performed in a different way.

The measurement quality was considered before, during, and after conducting the experiments, for example, with a written experimental design [26] and estimation of uncertainty in the position measurements of the antennas and the reflector. See [13] for further information on this.

\subsection{More Comparison of the Measurements with the Litera-} ture. The literature $[19,21,27]$ says that each noncoherent monochromatic source with a different DOA gives rise to a large eigenvalue, which is in accordance with our measurements C1S, C2SS, and U2SS.

The more uncorrelated the sources are the more equal in size the eigenvalues are in the experiments in $[19,21]$. In particular, uncorrelated sources with well separated DOAs give each an eigenvalue of similar size according to [21]. These statements are in accordance with our measurements I2SS and U2SS. In [25] a theoretic expression for the size of the two eigenvalues of two uncorrelated zero bandwidth signals is derived. In our measurements U2SS and U2SW the difference between the two largest eigenvalues for $1 \mathrm{SM}$ was about $6 \mathrm{~dB}$ larger than the prediction of the theory. The discrepancy could be due to a nonideal measurement system and to a nonzero bandwidth because of time limited measurements.

The result that the eigenspectrum starts with a single eigenvalue for (one or two) coherent transmitters for a single SM (a very short acquisition interval) in our measurements agrees with the result in [14] showing that the rank will be one for the case without motion and with zero jammer bandwidth and for the case with motion of radar and/or jammer, zero bandwidth, and a "vanishing short" acquisition interval. One of our results is that the number of large eigenvalues will increase up to a limit when the number of SMs is increased. This result is in agreement with the result in [14] showing that each scatterer will appear as an independent source when the acquisition interval goes to infinity. 


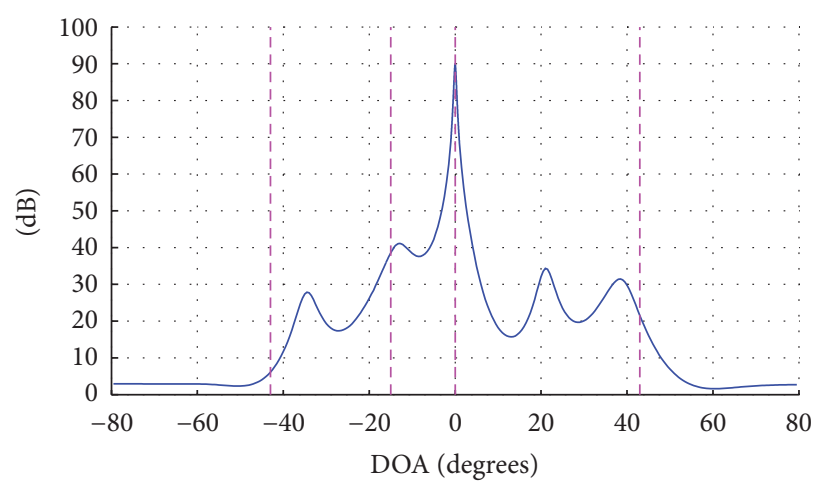

(a)
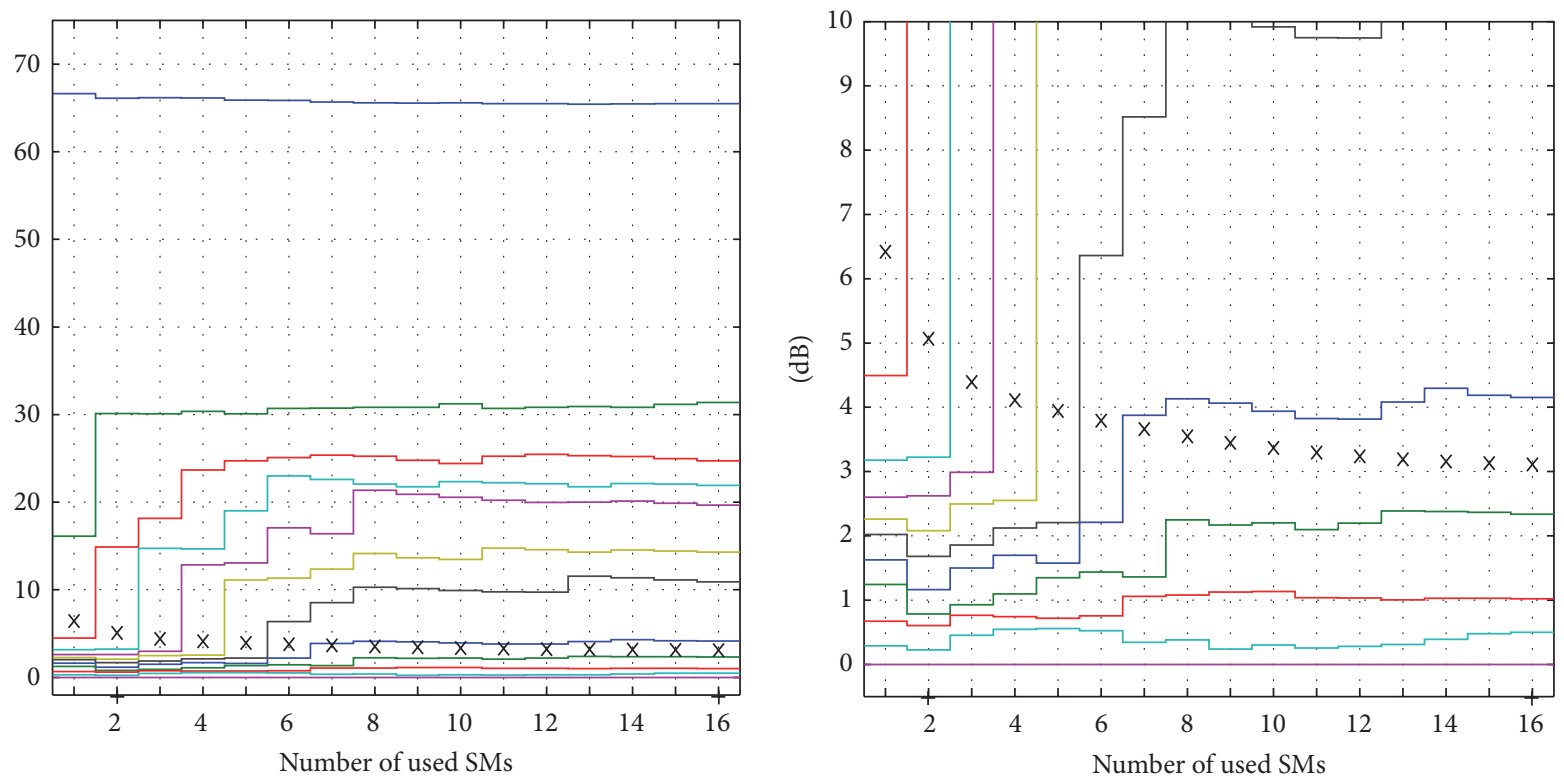

(b)

FiguRE 14: Measurement U2SW. Two uncorrelated transmitters, one strong and one weak. (a) Capon DOA spectrum. (b) Eigenspectrum. Zoom-in to the right. dB scale. See Section 3.3 for "SM."

We noticed by our measurements that the number of large eigenvalues depends on the signal power in comparison to the noise floor (compare measurement C1S in Figure 8 with C1W in Figure 12 and measurement U2SS with U2SW). The higher the signal power is, the more the eigenvalues of the spectrum tail will appear above the noise level. It is like an iceberg lifting above the ocean surface. This phenomenon is therefore called the iceberg effect. It is described in $[4,27]$ and there illustrated by simulations.

We found that the number of eigenpatterns which cover the reflection region (by the number of eigenpatterns which have their highest peak within the reflection region) is nearly independent of the number of used SMs. To estimate the number of signals using the eigenvalues we need many SMs but with the eigenpatterns it is enough with a few. Thus, it seems like the fact that the eigenpatterns are better for the estimation of the number of signals than the eigenvalues. Nevertheless, it is well-known that the number of large eigenvalues determines the required DoFs for interference suppression [4]. In [27] it is noticed, probably from simulations, that the eigenpatterns corresponding to the signal sources were "essentially unaffected" by a "modest amount" of interference subspace leakage, which is in agreement with our results.

We conclude that our measurement results agree in most cases with theoretic and simulated results presented in the literature.

\section{Conclusions}

We have designed an experiment for low-cost indoor measurements of direct and scattered signals with radar applications in mind. We have good control of the influencing factors, which is necessary to draw objective conclusions. The detailed description of our experiment could serve as a help for conducting other well-controlled experiments. Our experimental design has some characteristics:

(i) Emulation of coherent, intermediate correlated, and uncorrelated signal sources (Section 3.4). 

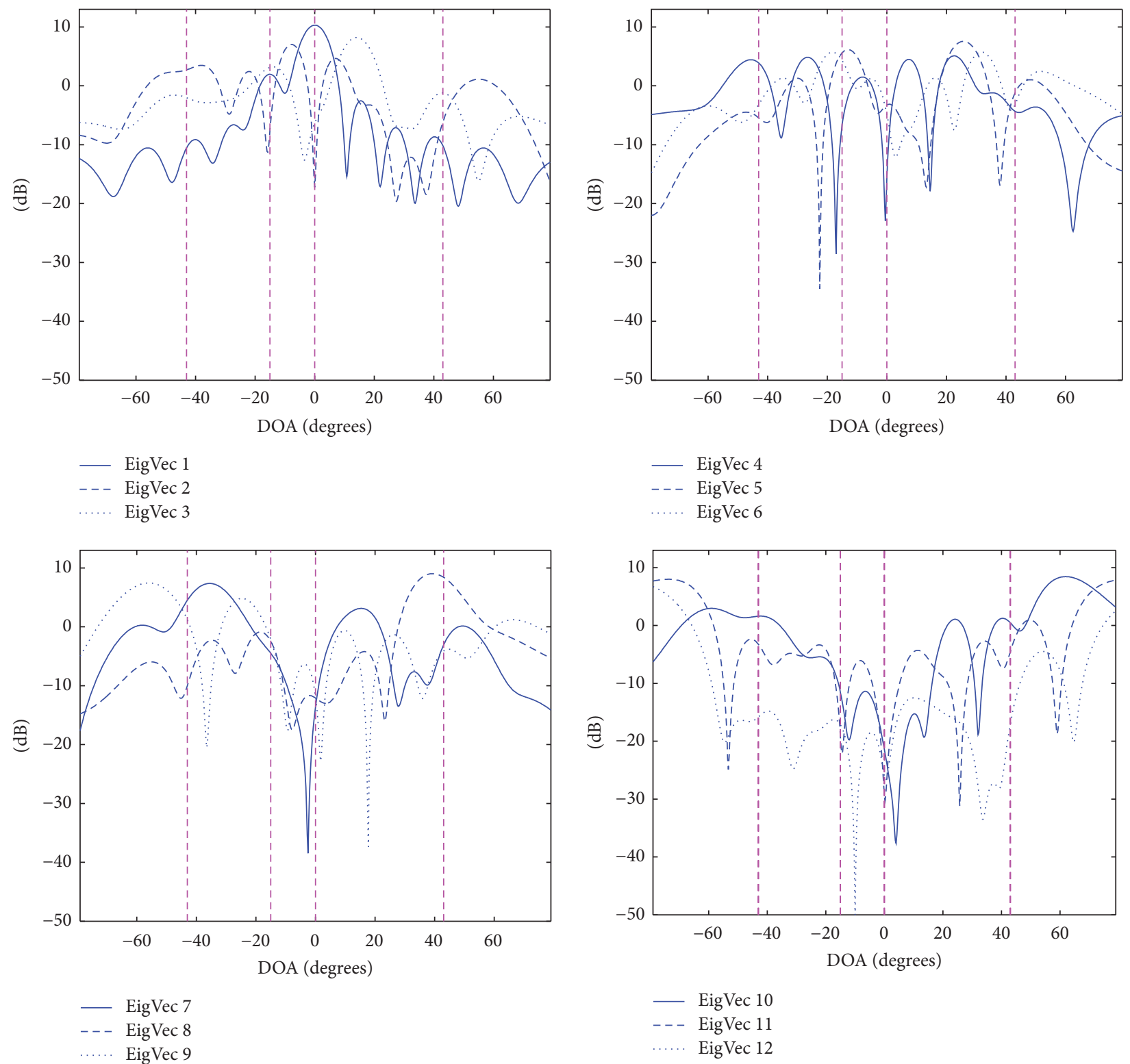

FIGURE 15: Eigenpatterns for measurement C2SS (two coherent strong transmitters). 1 SM. Dashed vertical lines are the true DOAs of the transmitter(s) and the nearest left and right corner of the reflector. Figure 6.32 in [13].

(ii) Calibration: when to calibrate and when not and also how to calibrate in different cases (Sections 3.2 and 3.5).

(iii) Near-field compensation: relation to receiving antenna properties, decorrelation, and eigenpatterns (Sections 3.4 and 3.5).

(iv) Noise eigenvalue spread: relation to calibration, hardware quality, and signal rank (Sections 2.1, 3.2, and 3.5).

(v) Emulation of a rough surface by a reflector (Section 3.3).

(vi) Decorrelation of the signals by movement of the reflector (Section 3.3). (vii) Acquisition interval for the estimation of the covariance matrix and its effects on the rank (Section 3.3).

(viii) Analysis methods: Capon DOA spectrum, eigenspectrum, and eigenpatterns (Section 3.5).

Section 4.3 summarizes our measured properties of direct and scattered signals. The agreement of our measured properties with theoretic and simulated results presented in the literature shows that our experimental design is realistic and sound.

\section{Competing Interests}

The authors declare that they have no competing interests. 

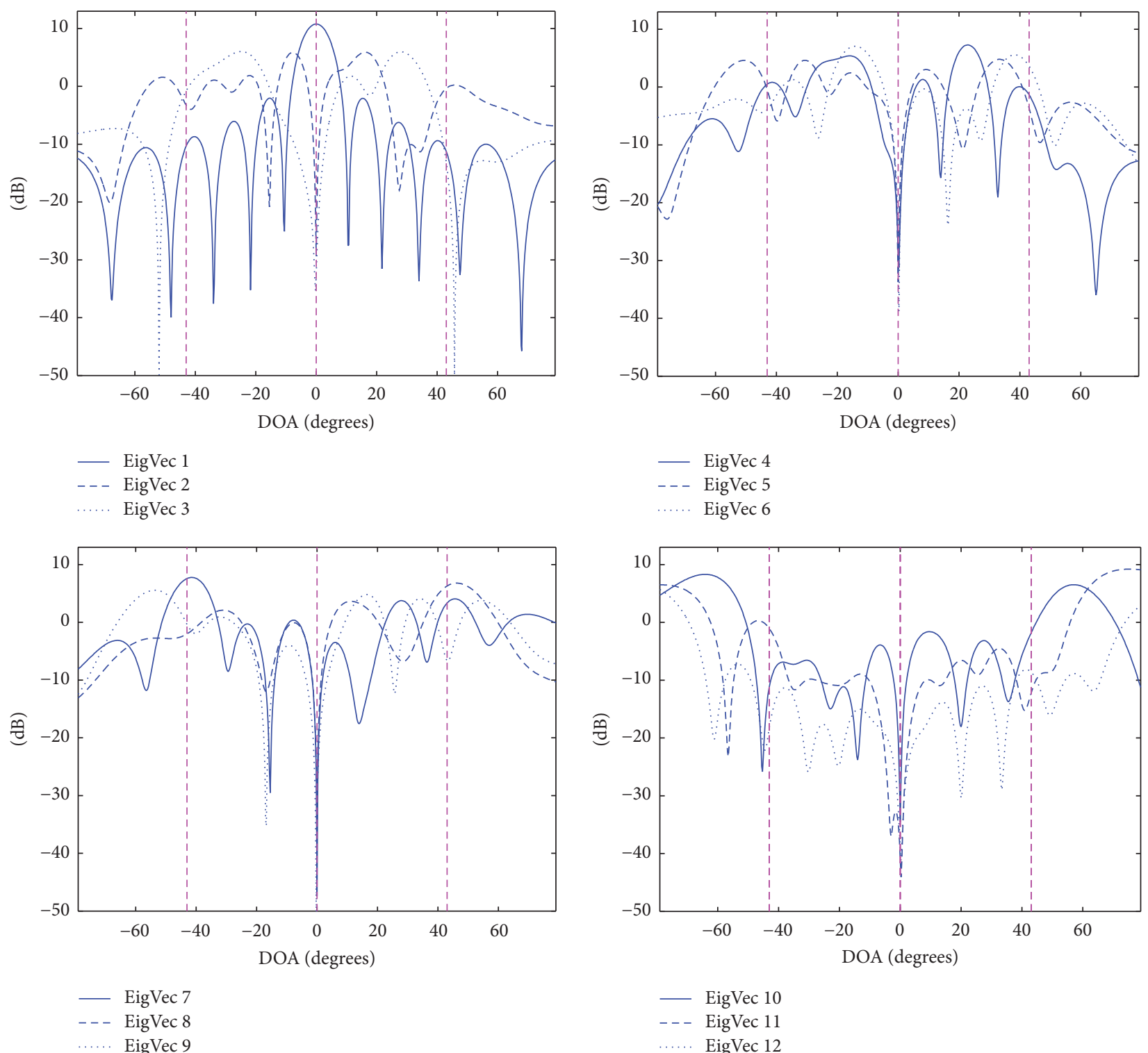

FIGURE 16: Eigenpatterns for measurement C1W (a single weak transmitter). 1 SM. Dashed vertical lines are the true DOAs of the transmitter(s) and the nearest left and right corner of the reflector. Figure 6.20 in [13].

\section{Acknowledgments}

This work has been financially supported by the Swedish Armed Forces, the Swedish Defence Materiel Administration, and the Swedish Knowledge Foundation.

\section{References}

[1] H. Krim and M. Viberg, "Two decades of array signal processing research: the parametric approach," IEEE Signal Processing Magazine, vol. 13, no. 4, pp. 67-94, 1996.

[2] I. S. Reed, J. D. Mallett, and L. E. Brennan, "Rapid convergence rate in adaptive arrays," IEEE Transactions on Aerospace and Electronic Systems, vol. 10, no. 6, pp. 853-863, 1974.

[3] L. E. Brennan and I. S. Reed, “Theory of adaptive radar," IEEE Transactions on Aerospace and Electronic Systems, vol. 9, no. 2, pp. 237-252, 1973.
[4] J. R. Guerci, Space-Time Adaptive Processing for Radar, Artech House, 2003.

[5] J. Ward, "Space-time adaptive processing for airborne radar," Tech. Rep. 1015, MIT Lincoln Laboratory, 1994.

[6] M. C. Wicks, M. Rangaswamy, and R. Adve, "Space-time adaptive processing: a knowledge-based perspective for airborne radar," IEEE Signal Processing Magazine, vol. 23, no. 1, pp. 5165,2006

[7] J. R. Guerci, J. S. Goldstein, and I. S. Reed, "Optimal and adaptive reduced-rank STAP," IEEE Transactions on Aerospace and Electronic Systems, vol. 36, no. 2, pp. 647-663, 2000.

[8] W. L. Melvin and M. E. Davis, "Adaptive cancellation method for geometry-induced nonstationary bistatic clutter environments," IEEE Transactions on Aerospace and Electronic Systems, vol. 43, no. 2, pp. 651-672, 2007. 
[9] M. Viberg, "Direction-of-arrival estimation," in Smart Antennas: State of the Art, chapter 16, Hindawi Publishing Corporation, New York, NY, USA, 2005.

[10] L. Pettersson, "An S-band digital beamforming antenna: design, procedures and performance," FOA Report FOA-R-99-01162408-SE, 1999.

[11] S. Björklund, P. Grahn, and A. Nelander, "Analysis of array antenna measurements with a rough surface reflector," in Proceedings of the 34th Asilomar Conference on Signals, Systems, and Computers, pp. 1135-1139, Pacific Grove, Calif, USA, November 2000.

[12] X. Mestre, "Improved estimation of eigenvalues and eigenvectors of covariance matrices using their sample estimates," IEEE Transactions on Information Theory, vol. 54, no. 11, pp. 51135129, 2008.

[13] S. Björklund, P. Grahn, A. Nelander, and A. Alm, "Hot clutter reduction in radar. Measurement report for anechoic chamber measurements in spring 2000," Tech. Rep. FOA-R-00-01806408-SE, 2000.

[14] P. M. Techau, J. R. Guerci, T. H. Slocumb, and L. J. Griffiths, "Performance bounds for hot and cold clutter mitigation," IEEE Transactions on Aerospace and Electronic Systems, vol. 35, no. 4, pp. 1253-1265, 1999.

[15] R. Cepeda, C. Vithanage, and W. Thompson, "From wideband to ultrawideband: channel diversity in low-mobility indoor environments," IEEE Transactions on Antennas and Propagation, vol. 59, no. 10, pp. 3882-3889, 2011.

[16] L. Pettersson, M. Danestig, and U. Sjostrom, "An experimental S-band digital beamforming antenna," IEEE Aerospace and Electronic Systems Magazine, vol. 12, no. 11, pp. 19-26, 1997.

[17] S. Björklund and A. Heydarkhan, "High resolution direction of arrival estimation methods applied to measurements from a digital array antenna," in Proceedings of the IEEE Sensor Array and Multichannel Signal Processing Workshop (SAM '00), pp. 464-468, Cambridge, Mass, USA, March 2000.

[18] E. M. Friel and K. M. Pasala, "Effects of mutual coupling on the performance of STAP antenna arrays," IEEE Transactions on Aerospace and Electronic Systems, vol. 36, no. 2, pp. 518-527, 2000.

[19] F. Haber and M. Zoltowski, "Spatial spectrum estimation in a coherent signal environment using an array in motion," IEEE Transactions on Antennas and Propagation, vol. AP-34, no. 3, pp. 301-310, 1986.

[20] C. A. Balanis, Antenna Theory. Analysis and Design, John Wiley \& Sons, New York, NY, USA, 2nd edition, 1997.

[21] A. Farina, Antenna-Based Signal Processing Techniques for Radar Systems, Artech House, Norwood, Mass, USA, 1992.

[22] J. Capon, "High-resolution frequency-wavenumber spectrum analysis," Proceedings of the IEEE, vol. 57, no. 8, pp. 1408-1418, 1969.

[23] W. F. Gabriel, "Using spectral estimation techniques in adaptive processing antenna systems," IEEE Transactions on Antennas and Propagation, vol. AP-34, no. 3, pp. 291-300, 1986.

[24] V. U. Reddy, A. Paulraj, and T. Kailath, "Performance analysis of the optimum beamformer in the presence of correlated sources and its behavior under spatial smoothing," IEEE Transactions on Acoustics, Speech, and Signal Processing, vol. 35, no. 7, pp. 927936, 1987.

[25] M. Zatman, "How narrow is narrowband?" IEE ProceedingsRadar, Sonar and Navigation, vol. 145, no. 2, pp. 85-91, 1998.
[26] S. Björklund, "Hot clutter reduction in radar. Experimental design for anechoic chamber measurements," Technical Report FOA Memo 00-3316/L, 2000.

[27] J. R. Guerci and J. S. Bergin, "Principal components, covariance matrix tapers, and the subspace leakage problem," IEEE Transactions on Aerospace and Electronic Systems, vol. 38, no. 1, pp. 152-162, 2002. 


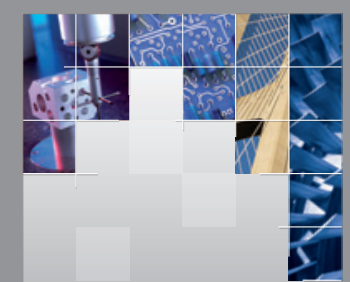

\section{Enfincering}
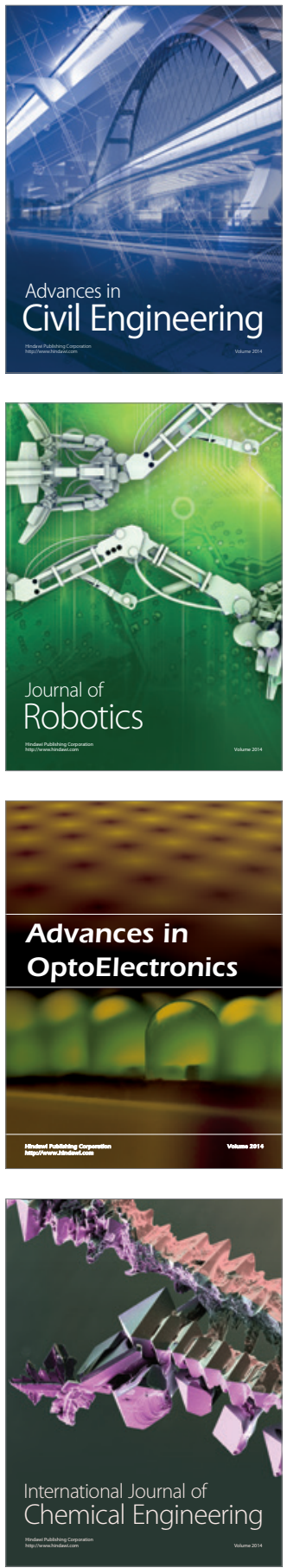

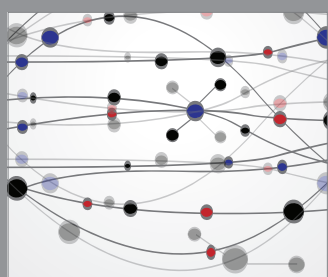

The Scientific World Journal

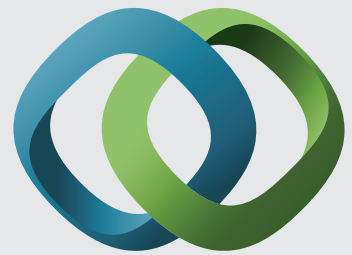

\section{Hindawi}

Submit your manuscripts at

http://www.hindawi.com
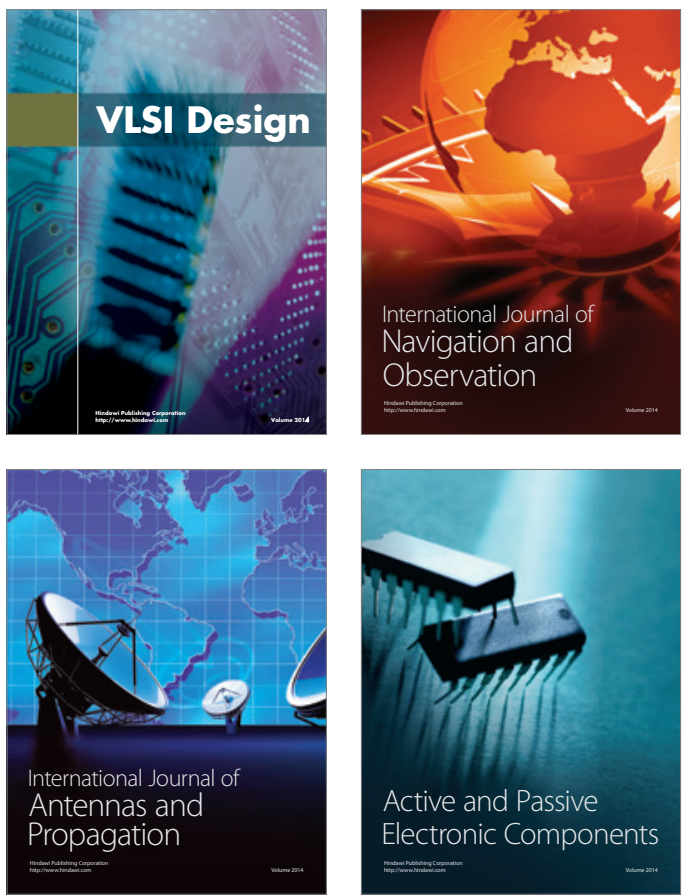
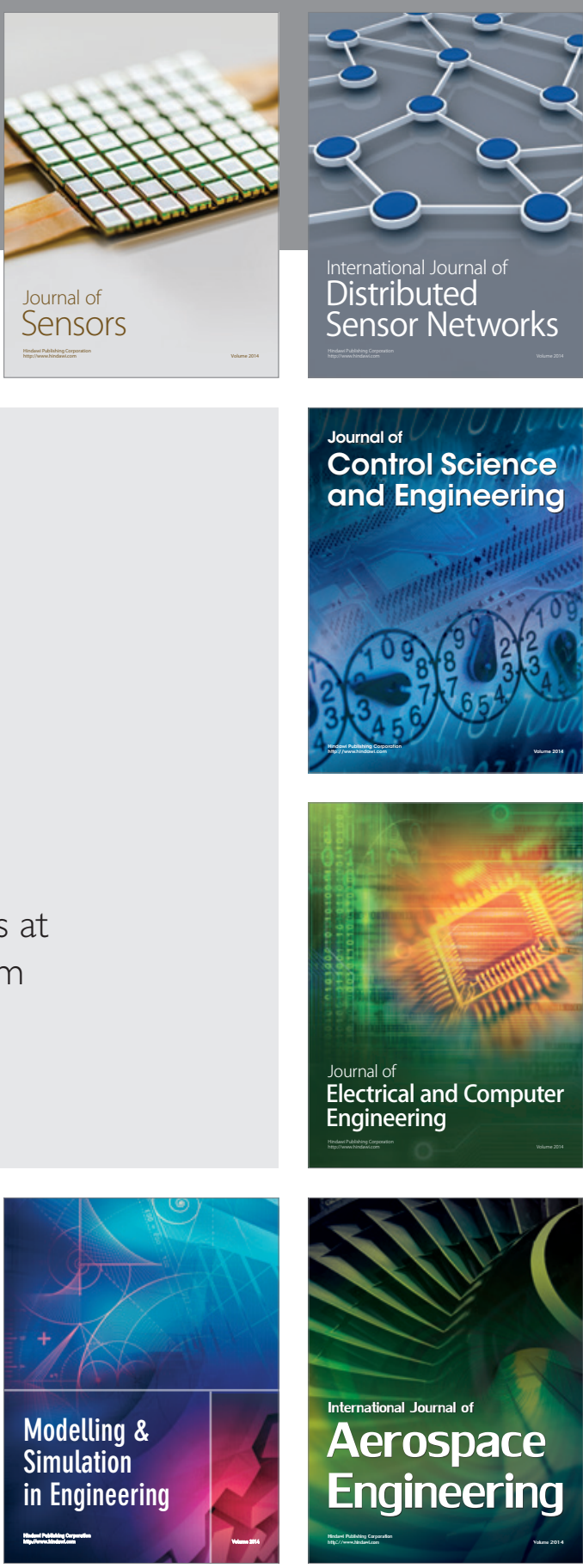

International Journal of

Distributed

Sensor Networks

Journal of

Control Science

and Engineering
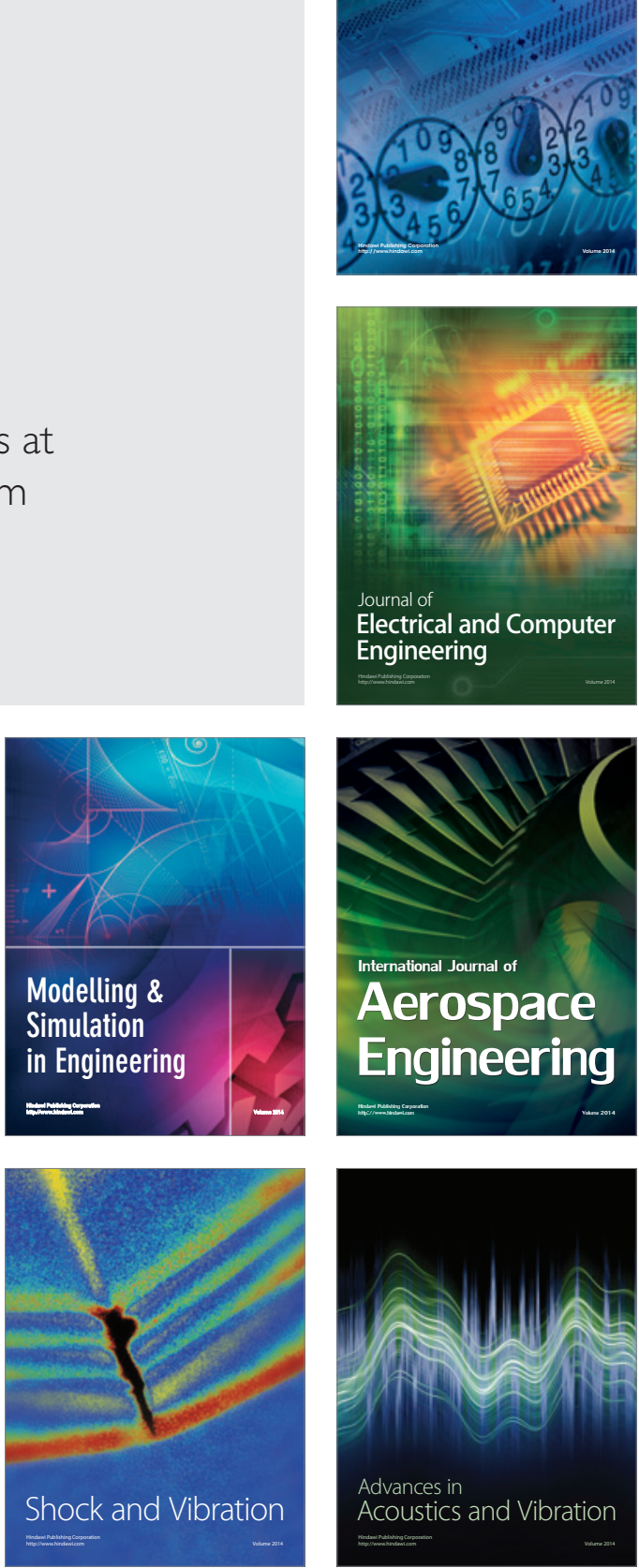